Supporting Information

\title{
Enantioselective Synthesis of (-)-cis-Clavicipitic acid
}

\author{
Jin-Mo Ku, ${ }^{a}$ Byeong-Seon Jeong, ${ }^{b}$ Sang-sup Jew, ${ }^{a, *}$ and Hyeung-geun Park ${ }^{a, *}$ \\ ${ }^{a}$ Research Institute of Pharmaceutical Science and College of Pharmacy, Seoul National \\ University, Seoul 151-742, Korea, and ${ }^{b}$ College of Pharmacy, Yeungnam University, Gyeongsan \\ 712-749, Korea
}

hgpk@plaza.snu.ac.kr

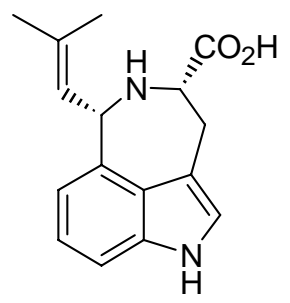

(-)-cis-Clavicipitic acid

\section{Contents}

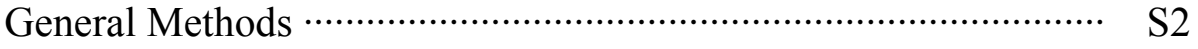

Experimental Section ….................................................. S2

${ }^{1} \mathrm{H} \&{ }^{13} \mathrm{C}$ NMR Spectra ……............................................. S13 


\section{General Methods.}

The ${ }^{1} \mathrm{H}-\mathrm{NMR}$ and ${ }^{13} \mathrm{C}-\mathrm{NMR}$ were recorded at $300 \mathrm{MHz}$ and $75 \mathrm{MHz}$, respectively. The chemical shifts were reported in ppm relative to $\mathrm{CHCl}_{3}(\delta 7.24)$ or $\mathrm{C}_{6} \mathrm{H}_{6}(\delta 7.16)$ for ${ }^{1} \mathrm{H}-\mathrm{NMR}$ and relative to the central $\mathrm{CDCl}_{3}(\delta 77.00)$ or $\mathrm{C}_{6} \mathrm{H}_{6}-d_{6}(\delta 129.39)$ resonance for ${ }^{13} \mathrm{C}-\mathrm{NMR}$. Optical rotations were measured on a digital polarimeter. Melting points were not corrected. Flash column chromatography was carried out using silica gel (230 400 mesh). The enantiomeric excess (ee) of the products was determined by HPLC using $4.6 \mathrm{~mm} \times 250 \mathrm{~mm}$ Chiralcel OD-H columns. All solvents and commercially available chemicals were used without additional purification. Dichloromethane was distilled from $\mathrm{CaH}_{2}$.

\section{Experimental Section}

\section{4-Iodo-1H-indole-3-carboxylic acid methyl ester (10)}

To a solution of $9(14.0 \mathrm{~g}, 80.0 \mathrm{mmol})$ in TFA $(560 \mathrm{~mL})$ under argon at room temperature was added thallium(III) trifluoroacetate $(65.0 \mathrm{~g}, 120.0 \mathrm{mmol})$, and the reaction mixture was stirred for 2 hours at room temperature. After TFA was evaporated in vacuo until a dark brown oil was obtained. This oil was suspended in $\mathrm{H}_{2} \mathrm{O}(910 \mathrm{~mL})$, KI $(39.7 \mathrm{~g}, 1.2 \mathrm{~mol})$ was added to this suspension. The reaction was stirred for 2 hours at room temperature. After dichloromethane:methanol $(95: 5, \mathrm{v} / \mathrm{v})$ was poured into the reaction mixture and the insoluble precipitates were filtered off through a plug of celite. The organic layer was separated and washed with aq. sodium thiosulfate and brine, dried over anhydrous $\mathrm{Na}_{2} \mathrm{SO}_{4}$. The excess solvent was removed in vacuo. The residue was purified by column chromatography on silica gel (hexanes:EtOAc $=6: 1$ and 2:1) to afford $10(17.8 \mathrm{~g}, 74.0 \%$ yield) as a yellowish solid.

mp. $125-126{ }^{\circ} \mathrm{C} .{ }^{1} \mathrm{H}$ NMR $\left(300 \mathrm{MHz}, \mathrm{CHCl}_{3}-d_{3}\right) \delta 8.87$ (bs, $\left.1 \mathrm{H}\right), 7.87(\mathrm{~d}, J=2.94 \mathrm{~Hz}, 1 \mathrm{H}), 7.82$ 
(dd, $J=0.93 \mathrm{~Hz}, 7.50 \mathrm{~Hz}, 1 \mathrm{H}), 7.40(\mathrm{dd}, J=0.93 \mathrm{~Hz}, 8.04 \mathrm{~Hz}, 1 \mathrm{H}), 6.93(\mathrm{t}, J=7.86 \mathrm{~Hz}, 1 \mathrm{H})$,

3.92(s, 3H) ppm; ${ }^{13} \mathrm{C}$ NMR $\left(100 \mathrm{MHz}, \mathrm{CHCl}_{3}-d_{3}\right) \delta 164.8,136.7,135.0,131.9,127.8,124.4$, 111.8, 110.5, 84.7, 51.3 ppm; IR (KBr) v 3294, 2944, 1691, 1524, 1440, 1407, 1344, 1254, 1163 , 1038, 937, 898, $761 \mathrm{~cm}^{-1}$; MS (FAB+): m/z $302[\mathrm{M}+\mathrm{H}]^{+}$; HRMS calculated for $\mathrm{C}_{10} \mathrm{H}_{9} \mathrm{INO}_{2}$ : 301.9678; Found: $301.9688[\mathrm{M}+\mathrm{H}]^{+}$.

\section{4-Iodo-indole-1, 3-dicarboxylic acid 1-tert-butyl ester 3-methyl ester (11)}

To a toluene solution $(82 \mathrm{~mL})$ of $\mathbf{1 0}(7.4 \mathrm{~g}, 24.5 \mathrm{mmol})$ was treated at room temperature with ditert-butyl dicarbonate $(5.9 \mathrm{~g}, 27.0 \mathrm{mmol})$ and dimethyl-pyridin-4-yl-amine (229 $\mathrm{mg}, 2.5 \mathrm{mmol})$ under argon. The reaction mixture was stirred at room temperature until starting material had been consumed $(1 \mathrm{~h})$. The reaction mixture was quenched with water, and diluted with ethyl acetate $(1 \mathrm{~L})$, washed with water $(200 \mathrm{~mL})$, dried over $\mathrm{Na}_{2} \mathrm{SO}_{4}$, filtered, and concentrated in vacuo. The residue was purified by column chromatography on silica gel (hexanes:EtOAc $=6: 1$ ) to afford 11 (12.1 g, 98.2 \% yield) as a yellowish amorphous compound.

${ }^{1} \mathrm{H}$ NMR $\left(300 \mathrm{MHz}, \mathrm{CHCl}_{3}-d_{3}\right) \delta 8.27(\mathrm{~d}, J=8.25,1 \mathrm{H}), 8.11(\mathrm{~s}, 1 \mathrm{H}), 7.83(\mathrm{dd}, J=0.93 \mathrm{~Hz}, 7.71$ $\mathrm{Hz}, 1 \mathrm{H}), 7.02$ (t, $J=8.04 \mathrm{~Hz}, 1 \mathrm{H}), 3.93(\mathrm{~s}, 3 \mathrm{H}), 1.65(\mathrm{~s}, 9 \mathrm{H}) \mathrm{ppm} ;{ }^{13} \mathrm{C} \mathrm{NMR}^{\left(100 \mathrm{MHz}, \mathrm{CHCl}_{3^{-}}\right.}$ $\left.d_{3}\right) \delta 164.1,148.3,136.2,135.9,131.4,129.5,126.2,115.2,114.7,85.4,84.8,51.8,28.0$ ppm; IR $(\mathrm{KBr}) \vee 2979,1742,1552,1414,1365,1258,1220,1151,1083,930,843,762 \mathrm{~cm}^{-1}$; MS (FAB+): m/z $402[\mathrm{M}]^{+}$; HRMS calculated for $\mathrm{C}_{15} \mathrm{H}_{17} \mathrm{NIO}_{4}$ : 402.0202 ; Found: $402.0189[\mathrm{M}]^{+}$.

\section{3-Hydroxymethyl-4-iodo-indole-1-carboxylic acid tert-butyl ester (12)}

To a stirred solution of $11(12.1 \mathrm{~g}, 90.2 \mathrm{mmol})$ in dichloromethane $(270 \mathrm{~mL})$ at $-78{ }^{\circ} \mathrm{C}$ was added 1 M DIBAL-H in dichloromethane $(66.4 \mathrm{~mL}, 66.4 \mathrm{mmol})$ dropwise via syringe, causing the clear 
solution to become a light yellowish color. The temperature was warmed to $0{ }^{\circ} \mathrm{C}$ and allowed to stir for 40 minutes. After the spot of the starting material on TLC was disappeared, the mixture was poured into aq. sodium potassium tartrate. Stirring for overnight followed the reaction. The solution was extracted with dichloromethane $(3 \times 500 \mathrm{~mL})$, and the organic layers were washed with water and brine, dried over $\mathrm{Na}_{2} \mathrm{SO}_{4}$, filtered, and concentrated in vacuo. The residue was purified by column chromatography on silica gel (hexanes:EtOAc $=6: 1)$ to afford $12(8.8 \mathrm{~g}$, $78.4 \%$ yield) as a white solid.

mp. $104.8{ }^{\circ} \mathrm{C},{ }^{1} \mathrm{H}$ NMR $\left(300 \mathrm{MHz}, \mathrm{CHCl}_{3}-d_{3}\right) \delta 8.25(\mathrm{~d}, J=8.25 \mathrm{~Hz}, 1 \mathrm{H}), 7.69(\mathrm{dd}, J=0.93 \mathrm{~Hz}$, $7.71 \mathrm{~Hz}, 1 \mathrm{H}), 7.69$ (s, 1H), 7.01 (t, $J=8.07 \mathrm{~Hz}, 1 \mathrm{H}), 4.99$ (dd, $J=0.9 \mathrm{~Hz}, 6.57 \mathrm{~Hz}, 1 \mathrm{H}), 2.13$ (t, $J$ $=6.6 \mathrm{~Hz}, 1 \mathrm{H}), 1.66(\mathrm{~s}, 9 \mathrm{H}) \mathrm{ppm} ;{ }^{13} \mathrm{C} \mathrm{NMR}\left(100 \mathrm{MHz}, \mathrm{CHCl}_{3}-d_{3}\right) \delta 149.3,137.2,134.3,131.3$, 127.0, 126.2, 121.3, 115.8, 84.8, 84.6, 56.8, 28.6 ppm; IR (KBr) v 3394, 2978, 1735, 1553, 1418, 1369, 1279, 1157, 1096, 1048, $771 \mathrm{~cm}^{-1}$; MS (FAB+): m/z $373[\mathrm{M}]^{+}$; HRMS calculated for $\mathrm{C}_{14} \mathrm{H}_{16} \mathrm{INO}_{3}: 373.0175$; Found: $373.0185[\mathrm{M}]^{+}$.

\section{3-Bromomethyl-4-iodo-indole-1-carboxylic acid tert-butyl ester (13)}

12 (496 mg, $1.3 \mathrm{mmol})$ in dichloromethane $(26.6 \mathrm{~mL})$ under argon at $-78{ }^{\circ} \mathrm{C}$ was treated with 1 $\mathrm{M} \mathrm{PBr}_{3}$ in dichloromethane $(1.6 \mathrm{~mL}, 1.6 \mathrm{mmol})$. The clear solution was warmed to $0{ }^{\circ} \mathrm{C}$ and allowed to stir for 40 minutes. After the spot of the SM on TLC was disappeared, the solution was quenched by aq. $\mathrm{NaHCO}_{3}$. The mixture was washed with water and brine. The organic layer was dried over anhydrous $\mathrm{Na}_{2} \mathrm{SO}_{4}$ and concentrated. The residue was purified by short column chromatography on silica gel (hexanes:EtOAc $=6: 1)$ to afford $\mathbf{1 3}(456.5 \mathrm{mg}, 80.3 \%$ yield) as clear, viscous oil.

${ }^{1} \mathrm{H}$ NMR $\left(300 \mathrm{MHz}, \mathrm{CHCl}_{3}-d_{3}\right) \delta 8.22(\mathrm{~d}, J=8.25 \mathrm{~Hz}, 1 \mathrm{H}), 7.76(\mathrm{~s}, 1 \mathrm{H}), 7.73(\mathrm{~d}, J=7.71 \mathrm{~Hz}$, 
1H), $7.00(\mathrm{t}, J=8.04 \mathrm{~Hz}, 1 \mathrm{H}), 4.92(\mathrm{~s}, 2 \mathrm{H}), 1.64(\mathrm{~s}, 9 \mathrm{H}) \mathrm{ppm} ;{ }^{13} \mathrm{C} \mathrm{NMR}\left(75 \mathrm{MHz}, \mathrm{CHCl}_{3}-d_{3}\right) \delta$ $148.5,136.4,134.9,129.1,128.3,126.1,118.1,115.4,84.7,84.2,28.1,25.3 \mathrm{ppm}$; IR (KBr) $v$ 2977, 1740, 1553, 1415, 1355, 1261, 1159, 1122, 1089, 1054, $841 \mathrm{~cm}^{-1}$; MS (FAB+): m/z 356 $[\mathrm{M}-\mathrm{Br}]^{+}$.

(S)-tert-Butyl-3-[3-tert-butoxy-2-(diphenylmethyleneamino)-3-oxopropyl]-4-iodo-1H-indole-

\section{1-carboxylate (14)}

To a mixture of $13(29 \mathrm{mg}, 0.067 \mathrm{mmol})$ and chiral catalyst $6(6.6 \mathrm{mg}, 0.0067 \mathrm{mmol})$ in solvent (dichloromethane:toluene $=3: 7)(0.67 \mathrm{~mL})$ was added $N$-(diphenylmethylene)glycine tert-butyl ester $(19.6 \mathrm{mg}, 0.067 \mathrm{mmol})$. The reaction mixture was then cooled $\left(0{ }^{\circ} \mathrm{C}\right)$, aq. $50 \% \mathrm{KOH}(13.7$ $\mathrm{mg}, 0.24 \mathrm{mmol}$ ) was added, and the reaction mixture was stirred at $0{ }^{\circ} \mathrm{C}$ until starting material had been consumed $(8 \mathrm{~h})$. The suspension was diluted with diethyl ether $(20 \mathrm{~mL})$, washed with water $(5 \mathrm{~mL} \times 2)$, dried over $\mathrm{Na}_{2} \mathrm{SO}_{4}$, filtered and concentrated in vacuo. Purification of residue by column chromatography on silica gel (hexanes:EtOAc $=10: 1$ ) afforded the desired product 14 (42 mg, $97 \%$ yield) as pale yellow oil. The enantioselectivity was determined by chiral HPLC analysis (DAICEL Chiralcel OD, hexanes:2-propanol $=500: 2.5$, flow rate $=1.0 \mathrm{~mL} / \mathrm{min}, 23^{\circ} \mathrm{C}$, $\lambda=254 \mathrm{~nm}$, retention times; $R$ (minor) $18.3 \mathrm{~min}, \mathrm{~S}$ (major) $23.1 \mathrm{~min}, 98.8 \%$ ee). $[\alpha]^{20}{ }_{\mathrm{D}}-185.7(c$ 1.00 , benzene).

${ }^{1} \mathrm{H}$ NMR $\left(300 \mathrm{MHz}, \mathrm{C}_{6} \mathrm{H}_{6}-d_{6}\right) \delta 8.57(\mathrm{~d}, J=8.25 \mathrm{~Hz}, 1 \mathrm{H}), 7.89-7.85(\mathrm{~m}, 2 \mathrm{H}), 7.63(\mathrm{~s}, 1 \mathrm{H}), 7.53$ (dd, $J=0.93 \mathrm{~Hz}, 7.71 \mathrm{~Hz}, 1 \mathrm{H}), 7.20-7.09(\mathrm{~m}, 3 \mathrm{H}), 6.99-6.93(\mathrm{~m}, 3 \mathrm{H}), 6.80-6.78(\mathrm{~m}, 2 \mathrm{H}), 6.69(\mathrm{t}$, $J=6.00 \mathrm{~Hz}, 1 \mathrm{H}), 4.94(\mathrm{dd}, J=4.02 \mathrm{~Hz}, 8.97 \mathrm{~Hz}, 1 \mathrm{H}), 4.25(\mathrm{dd}, J=3.48 \mathrm{~Hz}, 14.46 \mathrm{~Hz}, 1 \mathrm{H}), 3.61$ (dd, $J=9.15 \mathrm{~Hz}, 14.46 \mathrm{~Hz}, 1 \mathrm{H}), 1.40(\mathrm{~s}, 9 \mathrm{H}), 1.29(\mathrm{~s}, 9 \mathrm{H}) \mathrm{ppm} ;{ }^{13} \mathrm{C} \mathrm{NMR}\left(100 \mathrm{MHz}, \mathrm{C}_{6} \mathrm{H}_{6}-d_{6}\right) \delta$ $170.7,170.4,148.9,140.2,136.9,136.6,134.8,131.7,130.2,129.2,127.9,117.9,115.5,85.0$, 
83.2, 80.5, 66.8, 29.5, 28.1, 27.7 ppm; IR (KBr) v 2976, 1734, 1621, 1416, 1367, 1283, 1255, 1156, 1093, 1054, 845, 775, $699 \mathrm{~cm}^{-1}$; MS $(\mathrm{FAB}+) \mathrm{m} / \mathrm{z} 651[\mathrm{M}]^{+}$; HRMS calculated for $\mathrm{C}_{33} \mathrm{H}_{36} \mathrm{IN}_{2} \mathrm{O}_{4}$ : 651.1720; Found: $651.1724[\mathrm{M}]^{+}$.

(S,E)-tert-Butyl-3-[3-tert-butoxy-2-(diphenylmethyleneamino)-3-oxopropyl]-4-(3-hydroxy-3methylbut-1-enyl)-1H-indole-1-carboxylate (15)

To a mixture of $14(922 \mathrm{mg}, 1.4 \mathrm{mmol})$ and palladium acetate $(33.1 \mathrm{mg}, 0.14 \mathrm{mmol})$ in solvent (DMF: $\left.\mathrm{H}_{2} \mathrm{O}=1: 1\right)(5.7 \mathrm{~mL})$ was added $\mathrm{K}_{2} \mathrm{CO}_{3}(372 \mathrm{mg}, 2.8 \mathrm{mmol})$ at room temperature. 2Methyl-but-3-en-2-ol $(5.5 \mathrm{~g}, 63.8 \mathrm{mmol})$ was added to resulting solution, and the reaction mixture was stirred at $90{ }^{\circ} \mathrm{C}$ for $2 \mathrm{~h}$. After cooling, the suspension was diluted with diethyl ether $(200 \mathrm{~mL})$, washed with water $(10 \mathrm{~mL} \times 5)$, brine, dried over $\mathrm{Na}_{2} \mathrm{SO}_{4}$, filtered and concentrated in vacuo. Purification of residue by column chromatography on silica gel (hexanes:EtOAc $=6: 1$ ) afforded the desired product 15 (754 $\mathrm{mg}, 90 \%$ yield) as a brown crystalline. The enantioselectivity was determined by chiral HPLC analysis (DAICEL Chiralcel OD, hexanes:2propanol $=99: 1$, flow rate $=1.0 \mathrm{~mL} / \mathrm{min}, 23{ }^{\circ} \mathrm{C}, \lambda .=254 \mathrm{~nm}$, retention times; $S$ (major) $29.4 \mathrm{~min}$, $R$ (minor) $36.3 \mathrm{~min},>99 \% \mathrm{ee}$ ).

$[\alpha]^{25} \mathrm{D}-35.57(\mathrm{c} 1.00, \mathrm{EtOH}), \mathrm{mp} .133 .3{ }^{\circ} \mathrm{C} .{ }^{1} \mathrm{H}$ NMR $\left(300 \mathrm{MHz}, \mathrm{C}_{6} \mathrm{H}_{6}-d_{6}\right) \delta 8.55(\mathrm{~d}, J=8.25 \mathrm{~Hz}$, 1H), 7.73-7.69 (m, 2H), 7.33 (d, $J=15.54 \mathrm{~Hz}, 1 \mathrm{H}), 7.20(\mathrm{t}, J=7.68 \mathrm{~Hz}, 1 \mathrm{H}), 7.10(\mathrm{~d}, J=7.32$ Hz, 1H), 6.95-7.02 (m, 3H), $6.86(\mathrm{~d}, J=7.50 \mathrm{~Hz}, 1 \mathrm{H}), 6.77(\mathrm{t}, J=8.04 \mathrm{~Hz}, 2 \mathrm{H}), 6.40(\mathrm{~d}, J=6.96$ Hz, 2H), 5.77 (d, $J=15.57 \mathrm{~Hz}, 1 \mathrm{H}), 4.58(\mathrm{dd}, J=2.4 \mathrm{~Hz}, 10.44 \mathrm{~Hz}, 1 \mathrm{H}), 3.73$ (dd, $J=1.47 \mathrm{~Hz}$, $14.1 \mathrm{~Hz}, 1 \mathrm{H}) 3.54(\mathrm{dd}, J=10.44 \mathrm{~Hz}, 14.1 \mathrm{~Hz}, 1 \mathrm{H}), 2.57$ (s, 1H), $1.36(\mathrm{~s}, 12 \mathrm{H}), 1.25$ (s, 9H), 1.24 (s, 3H) ppm; ${ }^{13} \mathrm{C}$ NMR $\left(75 \mathrm{MHz}, \mathrm{C}_{6} \mathrm{H}_{6}-d_{6}\right) \delta 170.8,170.4,148.9,140.2,136.9,136.6,134.8$, 
$131.7,130.2,129.2,125.4,117.9,115.5,85.1,83.2,80.5,66.9,29.5,28.1,27.7$ ppm; IR (KBr) $v$ $2925,2855,1733,1423,1371,1286,1158,1097,971,759,699 \mathrm{~cm}^{-1} ; \mathrm{MS}(\mathrm{FAB}+) \mathrm{m} / \mathrm{z} 609[\mathrm{M}]^{+}$; HRMS calculated for $\mathrm{C}_{38} \mathrm{H}_{45} \mathrm{~N}_{2} \mathrm{O}_{5}: 609.3328$; Found: $609.3331[\mathrm{M}]^{+}$.

(S,E)-tert-Butyl-3-(2-amino-3-tert-butoxy-3-oxopropyl)-4-(3-hydroxy-3-methylbut-1-enyl)$1 H$-indole-1-carboxylate (16)

To a THF solution $(19 \mathrm{~mL})$ of $\mathbf{1 5}(792 \mathrm{mg}, 1.3 \mathrm{mmol})$ was added $0.1 \mathrm{M}$ citric acid (19.5 mL, 2.0 mmol) at room temperature. The reaction solution was followed to stir during overnight. The reaction mixture was quenched by sat. $\mathrm{NaHCO}_{3}(\mathrm{aq})$, and diluted with ethyl ether $(200 \mathrm{~mL})$. The organic layer was washed with water $(10 \mathrm{~mL} \times 3)$ and brine, dried over $\mathrm{Na}_{2} \mathrm{SO}_{4}$, filtered and concentrated in vacuo. Purification of residue by column chromatography on silica gel (hexanes:EtOAc $=6: 1$, EtOAc:MeOH $=20: 1)$ afforded the desired product $10(560 \mathrm{mg}, 97 \%$ yield) as pale oil.

$[\alpha]^{20}{ }_{\mathrm{D}}+16.8(c \mathrm{1} .00, \mathrm{MeOH}) .{ }^{1} \mathrm{H}$ NMR $\left(300 \mathrm{MHz}, \mathrm{MeOH}-d_{4}\right) \delta 8.07(\mathrm{dd}, J=2.4 \mathrm{~Hz}, 6.73 \mathrm{~Hz}$, 1H), $7.38(\mathrm{~s}, 1 \mathrm{H}), 7.17-7.23(\mathrm{~m}, 3 \mathrm{H}), 6.27$ (d, $J=15.75 \mathrm{~Hz}, 1 \mathrm{H}), 3.67(\mathrm{t}, J=7.35 \mathrm{~Hz}, 1 \mathrm{H}), 3.10$ (dd, $J=3.30 \mathrm{~Hz}, 7.89 \mathrm{~Hz}, 1 \mathrm{H}), 1.63$ (s, 9H), 1.42 (s, 6H), 1.29(s, 9H) ppm; ${ }^{13} \mathrm{C}$ NMR (100 MHz, $\left.\mathrm{MeOH}-d_{4}\right) \delta 176.1,151.4,142.9,138.4,134.0,129.4,127.1,126.3,126.0,122.8,118.9,115.9$, 85.5, 83.2, 72.4, 57.1, 35.1, 30.9, 30.8, 29.2, 28.9 ppm; IR (KBr) v 3365, 2975, 1730, 1423, 1373, 1287, 1255, 1159, 1095, 970, 849, $755 \mathrm{~cm}^{-1}$; MS (FAB+): m/z $445[\mathrm{M}]^{+}$; HRMS calculated for $\mathrm{C}_{25} \mathrm{H}_{37} \mathrm{~N}_{2} \mathrm{O}_{5}: 445.2702$; Found: $445.2708[\mathrm{M}]^{+}$.

3-(2-tert-Butoxycarbonyl-2-tert-butoxycarbonylamino-ethyl)-4-(3-hydroxy-3-methyl-but-1enyl)-indole-1-carboxylic acid tert-butyl ester (17) 
To a methylenechloride solution $(0.9 \mathrm{~mL})$ of $16(78 \mathrm{mg}, 17.6 \mathrm{mmol})$ was added di-tert-butyl dicarbonate $(5.9 \mathrm{~g}, 27.0 \mathrm{mmol})$ and triethylamine $(28 \mathrm{mg}, 28.0 \mathrm{mmol})$ at room temperature under argon. The reaction mixture was stirred at room temperature until starting material had been consumed $(2 \mathrm{~h})$. The reaction mixture was quenched with water, and diluted with ethyl acetate $(50 \mathrm{~mL})$, washed with water $(10 \mathrm{~mL})$, dried over $\mathrm{Na}_{2} \mathrm{SO}_{4}$, filtered, and concentrated in vacuo. The residue was purified by column chromatography on silica gel (hexanes:EtOAc $=4: 1$ ) to afford $\mathbf{1 7}$ (88.7 g, $93 \%$ yield) as a pale amorphous compound.

$[\alpha]^{20}{ }_{\mathrm{D}}-6.9\left(c\right.$ 1.00, EtOH). ${ }^{1} \mathrm{H}$ NMR $\left(300 \mathrm{MHz}, \mathrm{MeOH}-d_{4}\right) \delta 8.06(\mathrm{dd}, J=1.65 \mathrm{~Hz}, 7.5 \mathrm{~Hz}, 1 \mathrm{H})$, 7.39 (s, 1H), 7.27-7.17 (m, 3H), 6.29 (d, $J=15.75 \mathrm{~Hz}, 1 \mathrm{H}), 4.35$ (t, $J=7.89 \mathrm{~Hz}, 1 \mathrm{H}), 3.23-3.09$ $(\mathrm{m}, 2 \mathrm{H}), 1.63,9 \mathrm{H}), 1.43(\mathrm{~s}, 6 \mathrm{H}), 1.40(\mathrm{~s}, 9 \mathrm{H}), 1.28(\mathrm{~s}, 9 \mathrm{H}) \mathrm{ppm} ;{ }^{13} \mathrm{C}$ NMR $\left(100 \mathrm{MHz}, \mathrm{MeOH}-d_{4}\right)$ $\delta 174.0,158.5,151.5,142.9,138.4,134.0,129.4,127.2,126.3,126.0,122.9,118.5,115.9,85.6$, $83.5,81.4,72.5,57.0,32.2,30.6,29.5,29.2$, 28.9. ppm; IR (KBr) v 3438, 2977, 1732, 1505 , 1423, 1372, 1291, 1254, 1159, 1096, 969, 853, $757 \mathrm{~cm}^{-1}$; MS (FAB+): m/z $544[\mathrm{M}]^{+}$; HRMS calculated for $\mathrm{C}_{30} \mathrm{H}_{44} \mathrm{~N}_{2} \mathrm{O}_{7}: 544.3149$ Found: $544.3137[\mathrm{M}]^{+}$.

\section{$N, N$-di-Boc-clavicipitic acid tert-butyl ester (18)}

To a acetonitrile solution of $17(90 \mathrm{mg}, 0.16 \mathrm{mmol})$ was treated $\mathrm{PdCl}_{2}\left(\mathrm{CH}_{3} \mathrm{CN}\right)_{2}(4 \mathrm{mg}, 0.016$ mmol) at room temperature. The reaction solution was allowed to stir at room temperature until the starting material had been consumed. After cooling, the reaction mixture was quenched with water, and diluted with ethyl acetate, washed with water, dried over $\mathrm{Na}_{2} \mathrm{SO}_{4}$, filtered, and concentrated in vacuo. The residue was purified by column chromatography on silica gel (hexanes:EtOAc $=6: 1)$ to afford no separable a mixture of diastereomer $(66 \mathrm{mg}, 76 \%$ yield), cis18 and trans-18 (5:1), which was directly adapted to the next hydrolysis step. 


\section{Clavicipitic acid (1)}

A stirred solution of $18(83 \mathrm{mg}, 0.16 \mathrm{mmol})$ in dichloromethane $(3 \mathrm{~mL})$ was treated with $\mathrm{ZnBr}_{2}$ (213 mg, $0.96 \mathrm{mmol}, 6 \mathrm{eq})$ at room temperature in the presence of Ar. The resulting solution was allowed to stir until 18 were consumed $(6$ h). The reaction mixture was treated with water $(20$ $\mathrm{mL}$ ), and stirred again for $2 \mathrm{~h}$. After the organic layer was removed, the reaction mixture was directly subjected to ODS-silica gel column chromatography by gradient elution from $\mathrm{H}_{2} \mathrm{O}$ to MeOH. 1 was obtained at the fraction of $\mathrm{H}_{2} \mathrm{O} / \mathrm{MeOH}(2: 1)(38 \mathrm{mg}, 90 \%)$ as a diastereomeric mixture (cis : trans $=5: 1$ ). The diastereomers were separated by preparative TLC (silica $\mathrm{GF}_{254}$, $\mathrm{CHCl}_{3}: \mathrm{CH}_{3} \mathrm{OH}: \mathrm{c}-\mathrm{NH}_{4} \mathrm{OH}=80: 20: 1$, developed twice) to afford cis-1 (32 mg, 75\%) and trans-1 (6 mg, 15\%).

\section{(-)-trans-clavicipitic acid}

$\mathrm{R}_{\mathrm{f}} 0.28$, mp. $200-210{ }^{\circ} \mathrm{C}$ (dec.), $[\alpha]^{25} \mathrm{D}-171.5$ (c 0.16, EtOH). ${ }^{1} \mathrm{H}$ NMR (300 MHz, MeOH-d 4 ) $\delta$ $7.32(\mathrm{~d}, J=9.69 \mathrm{~Hz}, 1 \mathrm{H}), 7.19(\mathrm{~s}, 1 \mathrm{H}), 6.81(\mathrm{~d}, J=7.50 \mathrm{~Hz}, 1 \mathrm{H}), 5.57(\mathrm{~d}, J=8.40 \mathrm{~Hz}, 1 \mathrm{H}), 5.43$ $(\mathrm{d}, J=9.69 \mathrm{~Hz}, 1 \mathrm{H}), 3.97$ (d, $J=11.52 \mathrm{~Hz}, 1 \mathrm{H}), 3.79$ (d, $J=16.47 \mathrm{~Hz}, 1 \mathrm{H}), 3.19-3.10(\mathrm{~m}, 1 \mathrm{H})$, $1.96(\mathrm{~d}, J=1.29 \mathrm{~Hz}, 3 \mathrm{H}), 1.93(\mathrm{~d}, J=1.47 \mathrm{~Hz}, 3 \mathrm{H}) \mathrm{ppm} ;{ }^{13} \mathrm{C} \mathrm{NMR}\left(75 \mathrm{MHz}, \mathrm{MeOH}-d_{4}\right) \delta 124.2$, $122,5,122.3,119.0,112.6,65.2,61.4,28.9,26.0,18.5 \mathrm{ppm}$ (The other additional peaks were not detected because of its low solubility. In acidic or basic NMR solvent, trans-1 was decomposed.); IR $(\mathrm{KBr}) \vee 3419,1637,1428,1088,965,785,750,566 \mathrm{~cm}^{-1}$; MS (FAB+) m/z $271[\mathrm{M}]^{+}$; HRMS calculated for $\mathrm{C}_{16} \mathrm{H}_{18} \mathrm{~N}_{2} \mathrm{O}_{2}$ : 271.1447; Found: $271.1447[\mathrm{M}]^{+}$.

\section{(-)-cis-Clavicipitic acid}


$\mathrm{R}_{\mathrm{f}} 0.31, \mathrm{mp} .256{ }^{\circ} \mathrm{C}(\mathrm{dec}),.[\alpha]^{25}{ }_{\mathrm{D}}-238.0\left(\right.$ c 0.2, EtOH); ${ }^{1} \mathrm{H}$ NMR $\left(300 \mathrm{MHz}, \mathrm{MeOH}-d_{4}\right) \delta 7.33$ (d, $J=8.04 \mathrm{~Hz}, 1 \mathrm{H}), 7.21(\mathrm{~s}, 1 \mathrm{H}), 7.09(\mathrm{t}, J=7.89 \mathrm{~Hz}, 1 \mathrm{H}), 6.83$ (d, $J=7.50 \mathrm{~Hz}, 1 \mathrm{H}), 5.91(\mathrm{~d}, J$ $=8.76 \mathrm{~Hz}, 1 \mathrm{H}), 5.48(\mathrm{~d}, J=8.79 \mathrm{~Hz}, 1 \mathrm{H}), 4.19(\mathrm{dd}, J=3.84 \mathrm{~Hz}, 12.45 \mathrm{~Hz}, 1 \mathrm{H}), 3.72(\mathrm{dd}, J=$ $3.48 \mathrm{~Hz}, 16.47 \mathrm{~Hz}, 1 \mathrm{H}), 3.45-3.34$ (m, 1H), 1.93 (s, 3H), 1.88 (s, 3H) ppm; IR (KBr) v 3437, $1625,1383,1327,1258,1051,783,749,601 \mathrm{~cm}^{-1}$; MS (FAB+) m/z $271[\mathrm{M}]^{+}$; HRMS calculated for $\mathrm{C}_{16} \mathrm{H}_{18} \mathrm{~N}_{2} \mathrm{O}_{2}: 271.1447$; Found: $271.1448[\mathrm{M}]^{+}$. The ${ }^{13} C$ NMR spectroscopic data could not be obtained because of too low solubility in any organic neutral NMR solvent. In acidic or basic NMR solvent, cis-1 was decomposed.

Aminocyclization of 12 in acidic condition using PPTS and deprotection of $\mathrm{N}$-Boc and tertbutyl ester of 19 without epimerization of $\mathrm{C}(10)$ using $\mathrm{ZnBr}_{2}$.
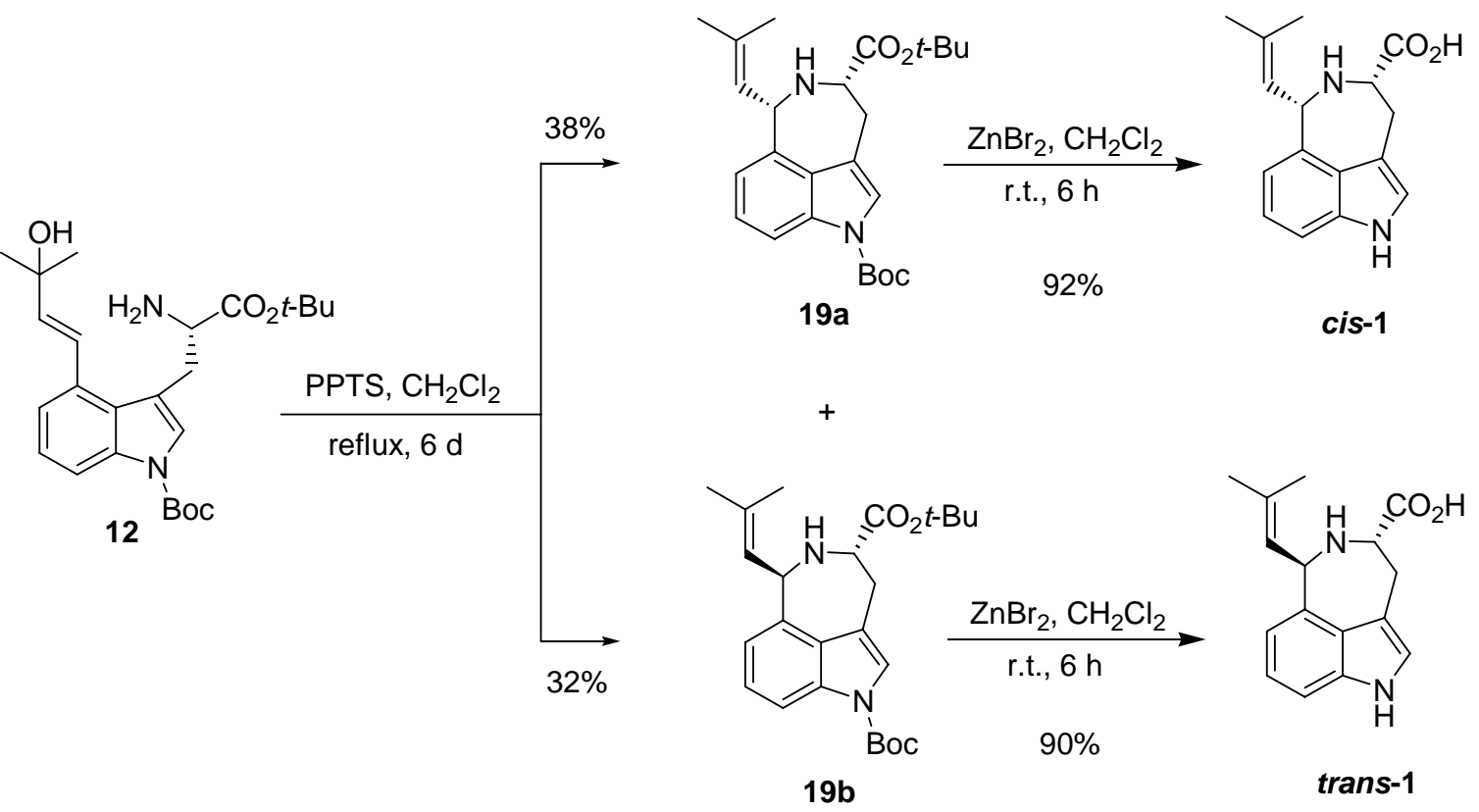


\section{(5S)-N-Boc-Clavicipitic acid tert-butyl ester (19a, 19b)}

To 12 (191 mg, $0.43 \mathrm{mmol})$ in dichloromethane $(43 \mathrm{~mL})$ was added PPTS (108.0 mL, 0.43 mmol) at room temperature. The resulting solution was allowed to stir at $60{ }^{\circ} \mathrm{C}$ under argon atmosphere for 6 days. The reaction mixture was quenched by saturated $\mathrm{NaHCO}_{3}$, and diluted with ethyl ether $(100 \mathrm{~mL})$. The organic layer was washed with water $(10 \mathrm{~mL} \times 3)$ and brine, dried over $\mathrm{Na}_{2} \mathrm{SO}_{4}$, filtered and concentrated in vacuo. Purification of the residue by column chromatography on silica gel (hexanes:EtOAc $=10: 1$ ) afforded the separable two diastereomer products (19a, 19b).

19a (cis-isomer) $\left(70 \mathrm{mg}, 38 \%\right.$ yield) as a sticky oil. $[\alpha]^{25}{ }_{\mathrm{D}}-86.3(c 1.00, \mathrm{EtOH}){ }^{1} \mathrm{H}$ NMR $(300$ $\left.\mathrm{MHz}, \mathrm{MeOH}-d_{4}\right) \delta 8.04(\mathrm{~d}, J=8.25 \mathrm{~Hz}, 1 \mathrm{H}), 7.46(\mathrm{~s}, 1 \mathrm{H}), 7.18(\mathrm{t}, J=7.68 \mathrm{~Hz}, 1 \mathrm{H}), 6.93(\mathrm{dt}, J=$ $1.08 \mathrm{~Hz}, 7.71 \mathrm{~Hz}, 1 \mathrm{H}), 5.44(\mathrm{dt}, J=1.29 \mathrm{~Hz}, 8.97 \mathrm{~Hz}, 1 \mathrm{H}), 4.85$ (d, $J=8.79 \mathrm{~Hz}, 1 \mathrm{H}), 3.68$ (dd, $J$ $=2.76 \mathrm{~Hz}, 11.52 \mathrm{~Hz}, 1 \mathrm{H}), 3.48(\mathrm{ddd}, J=1.08 \mathrm{~Hz}, 2.91,15.93 \mathrm{~Hz}, 1 \mathrm{H}), 2.92$ (ddd, $J=1.81 \mathrm{~Hz}$, $11.21 \mathrm{~Hz}, 15.75 \mathrm{~Hz}, 1 \mathrm{H}), 1.90(\mathrm{~d}, J=1.29,3 \mathrm{H}), 1.84(\mathrm{~d}, J=1.29,3 \mathrm{H}), 1.66(\mathrm{~s}, 9 \mathrm{H}), 1.52(\mathrm{~s}, 9 \mathrm{H})$ ppm; ${ }^{13} \mathrm{C}$ NMR (100 MHz, MeOH-d $) \delta 174.6,151.7,139.1,138.5,137.8,130.1,128.6,125.7$, $124.9,123.0,119.7,115.3,85.6,84.2,63.6,62.7,34.2,29.2,29.1,26.7,19.2$ ppm; IR (KBr) v 2976, 2923, 1729, 1424, 1379, 1283, 1379, 1283, 1157, 1094, 854, $759 \mathrm{~cm}^{-1}$; MS (FAB) m/z 427 $[\mathrm{M}]^{+}$; HRMS calculated for $\mathrm{C}_{25} \mathrm{H}_{34} \mathrm{~N}_{2} \mathrm{O}_{4}$ : 427.2597; Found: $427.2601[\mathrm{M}]^{+}$.

19b (trans-isomer) (57.7 mg, $32 \%$ yield) as a sticky oil.

$[\alpha]^{25}{ }_{\mathrm{D}}-47.6(c \mathrm{1.00}, \mathrm{EtOH}) ;{ }^{1} \mathrm{H}$ NMR $\left(300 \mathrm{MHz}, \mathrm{MeOH}-\mathrm{d}_{4}\right) \delta 7.96(\mathrm{~d}, J=8.28 \mathrm{~Hz}, 1 \mathrm{H}), 7.43(\mathrm{~d}$, $J=1.05 \mathrm{~Hz}, 1 \mathrm{H}), 7.16(\mathrm{t}, J=7.50 \mathrm{~Hz}, 1 \mathrm{H}), 6.89(\mathrm{dt}, J=0.93 \mathrm{~Hz}, 7.50 \mathrm{~Hz}, 1 \mathrm{H}), 5.40(\mathrm{dd}, J=1.29$ $\mathrm{Hz}, 2.76 \mathrm{~Hz}, 1 \mathrm{H}), 5.30$ (d, $J=8.58 \mathrm{~Hz}, 1 \mathrm{H}), 3.94$ (dd, $J=4.02 \mathrm{~Hz}, 11.16 \mathrm{~Hz}, 1 \mathrm{H}), 3.34$ (ddd, $J=$ $0.72 \mathrm{~Hz}, 3.84 \mathrm{~Hz}, 15.57 \mathrm{~Hz}, 1 \mathrm{H}), 3.13$ (ddd, $J=1.83 \mathrm{~Hz}, 11.19 \mathrm{~Hz}, 15.57 \mathrm{~Hz}, 1 \mathrm{H}), 1.83$ (d, $J=$ 
$1.08 \mathrm{~Hz}, 3 \mathrm{H}), 1.81(\mathrm{~d}, J=1.29 \mathrm{~Hz}, 3 \mathrm{H}), 1.65(\mathrm{~s}, 9 \mathrm{H}), 1.49$ (s, 9H) ppm; ${ }^{13} \mathrm{C}$ NMR $(75 \mathrm{MHz}$, $\left.\mathrm{MeOH}-d_{4}\right) \delta 173.0,150.0,138.2,136.2,134.7,128.3,125.3,123.8,122.1,120.4,117.9,123.0$, 83.3, 81.5, 56.8, 55.4, 30.6, 27.0, 26.9, 24.7, 17.8 ppm; IR (KBr) v 2975, 2927, 1730, 1428, 1385, 1283, 1253, 1155, 1102, 852, $754 \mathrm{~cm}^{-1}$; MS (FAB) $\mathrm{m} / \mathrm{z} 427[\mathrm{M}]^{+}$; HRMS calculated for $\mathrm{C}_{25} \mathrm{H}_{34} \mathrm{~N}_{2} \mathrm{O}_{4}: 427.2597$; Found: $427.2601[\mathrm{M}]^{+}$.

\section{(-)-cis-Clavicipitic acid (cis-1)}

A stirred solution of 19a $(18 \mathrm{mg}, 0.042 \mathrm{mmol})$ in dichloromethane $(0.8 \mathrm{~mL})$ was treated with $\mathrm{ZnBr}_{2}(38 \mathrm{mg}, 0.17 \mathrm{mmol})$ at room temperature. The resulting mixture was stirred for $6 \mathrm{~h}$, treated with water $(2 \mathrm{~mL})$, and stirred again for $2 \mathrm{~h}$. After the organic layer was removed, the reaction mixture was directly subjected to ODS-silica gel column chromatography by gradient elution from $\mathrm{H}_{2} \mathrm{O}$ to $\mathrm{MeOH}$. The product (cis-1) was obtained at the fraction of $\mathrm{H}_{2} \mathrm{O}: \mathrm{MeOH}(2: 1),(10.5$ mg, $92 \%)$ as a white solid.

\section{(-)-trans-Clavicipitic acid (trans-1)}

A stirred solution of $19 \mathbf{b}(37 \mathrm{mg}, 0.087 \mathrm{mmol})$ in $\mathrm{CH}_{2} \mathrm{Cl}_{2}(1.7 \mathrm{~mL})$ was treated with $\mathrm{ZnBr}_{2}(78$ $\mathrm{mg}, 0.35 \mathrm{mmol}$ ) at room temperature. The resulting mixture was stirred for $6 \mathrm{~h}$, treated with water $(3 \mathrm{~mL})$, and stirred again for $2 \mathrm{~h}$. After the organic layer was removed, the reaction mixture was directly subjected to ODS-silica gel column chromatography by gradient elution from $\mathrm{H}_{2} \mathrm{O}$ to $\mathrm{MeOH}$. The product (trans-1) was obtained at the fraction of $\mathrm{H}_{2} \mathrm{O}: \mathrm{MeOH}(2: 1),(21.0 \mathrm{mg}$, $90 \%)$ as a pale yellow solid. 


\section{Compound 10}

${ }^{1} \mathrm{H}$ NMR $\left(300 \mathrm{MHz}, \mathrm{CDCl}_{3}\right.$ )

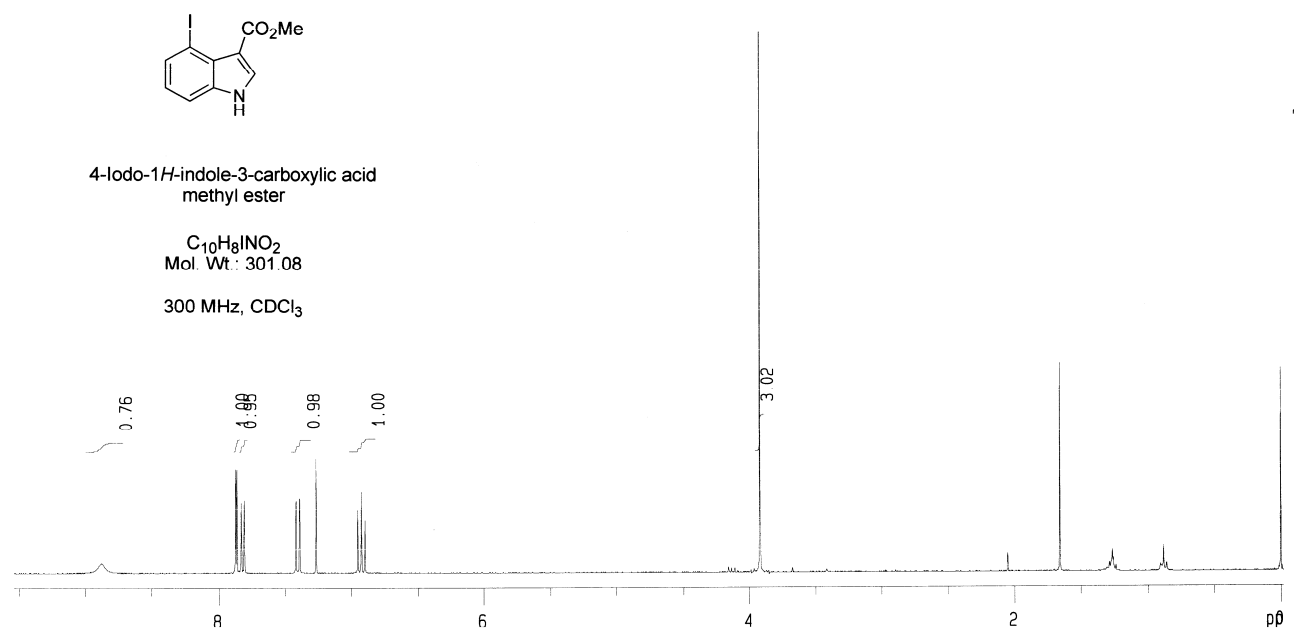

${ }^{13} \mathrm{C}$ NMR $\left(100 \mathrm{MHz}, \mathrm{CDCl}_{3}\right)$

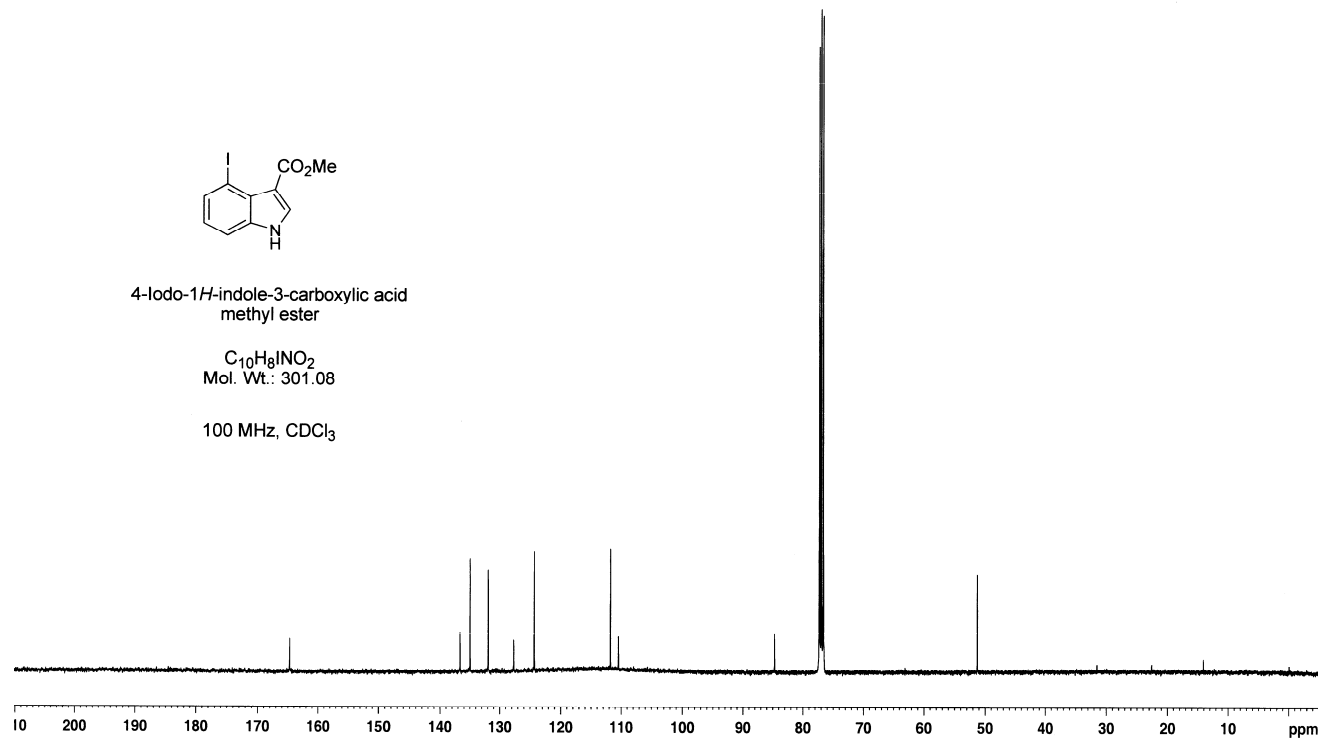

S13 


\section{Compound 11}

${ }^{1} \mathrm{H}$ NMR $\left(300 \mathrm{MHz}, \mathrm{CDCl}_{3}\right)$

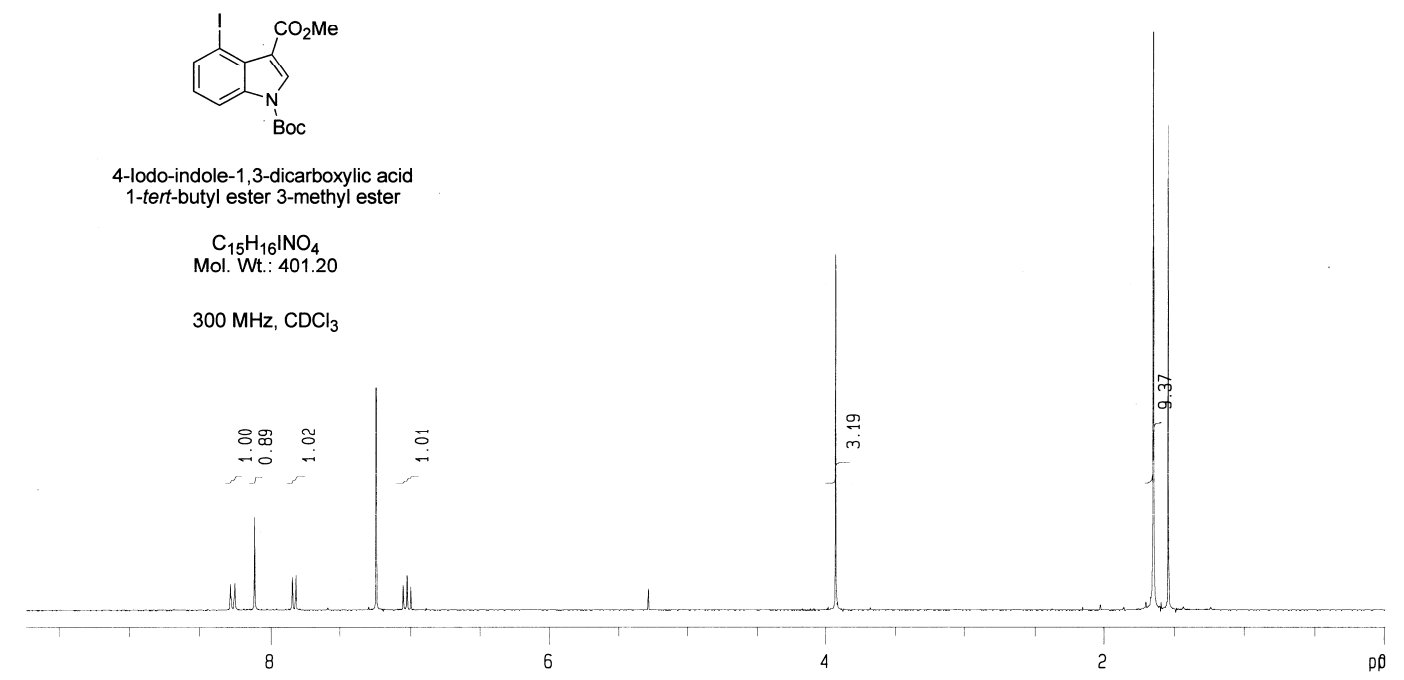

\section{${ }^{13} \mathrm{C}$ NMR $\left(100 \mathrm{MHz}, \mathrm{CDCl}_{3}\right)$}

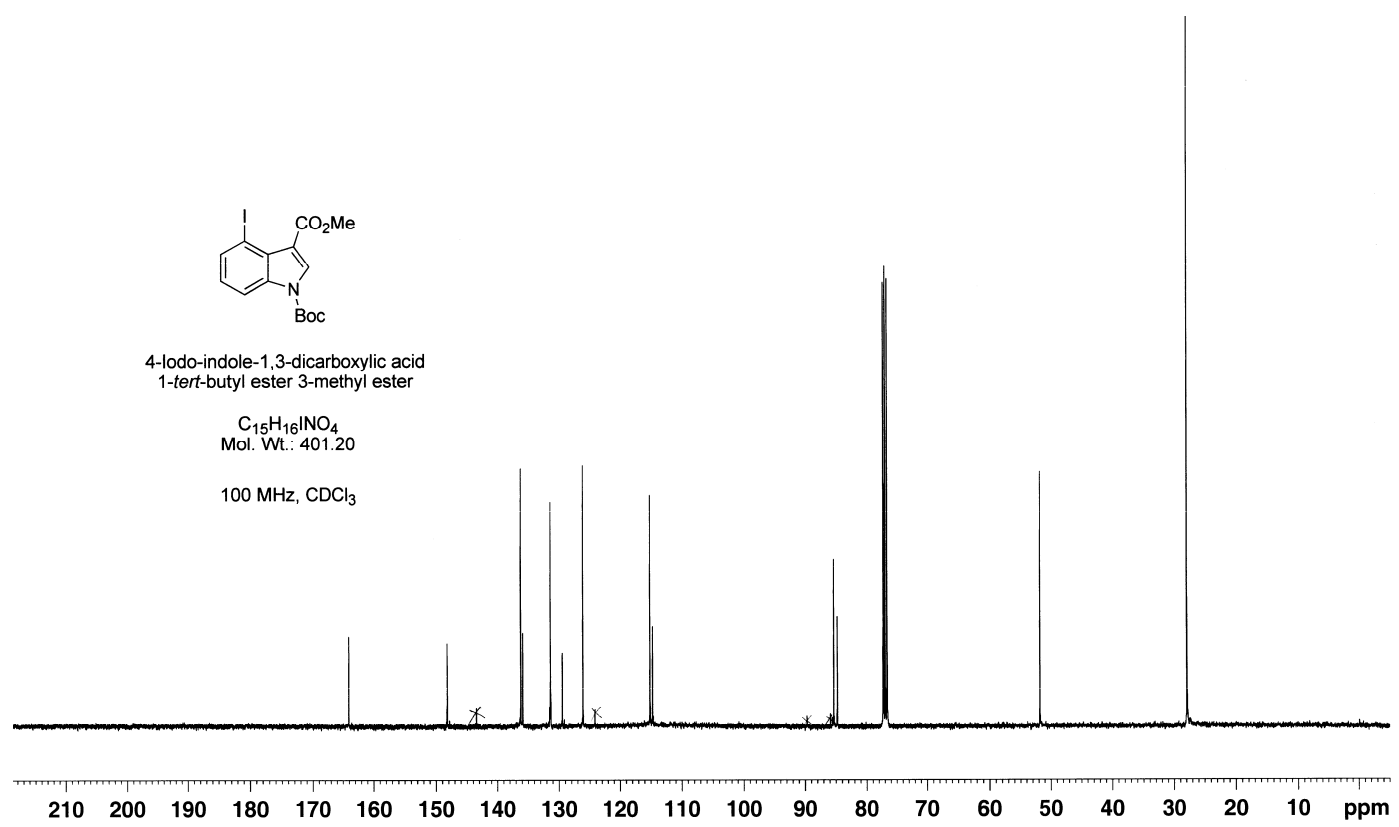




\section{Compound 12}

${ }^{1} \mathrm{H}$ NMR (300 MHz, $\mathrm{CDCl}_{3}$ )

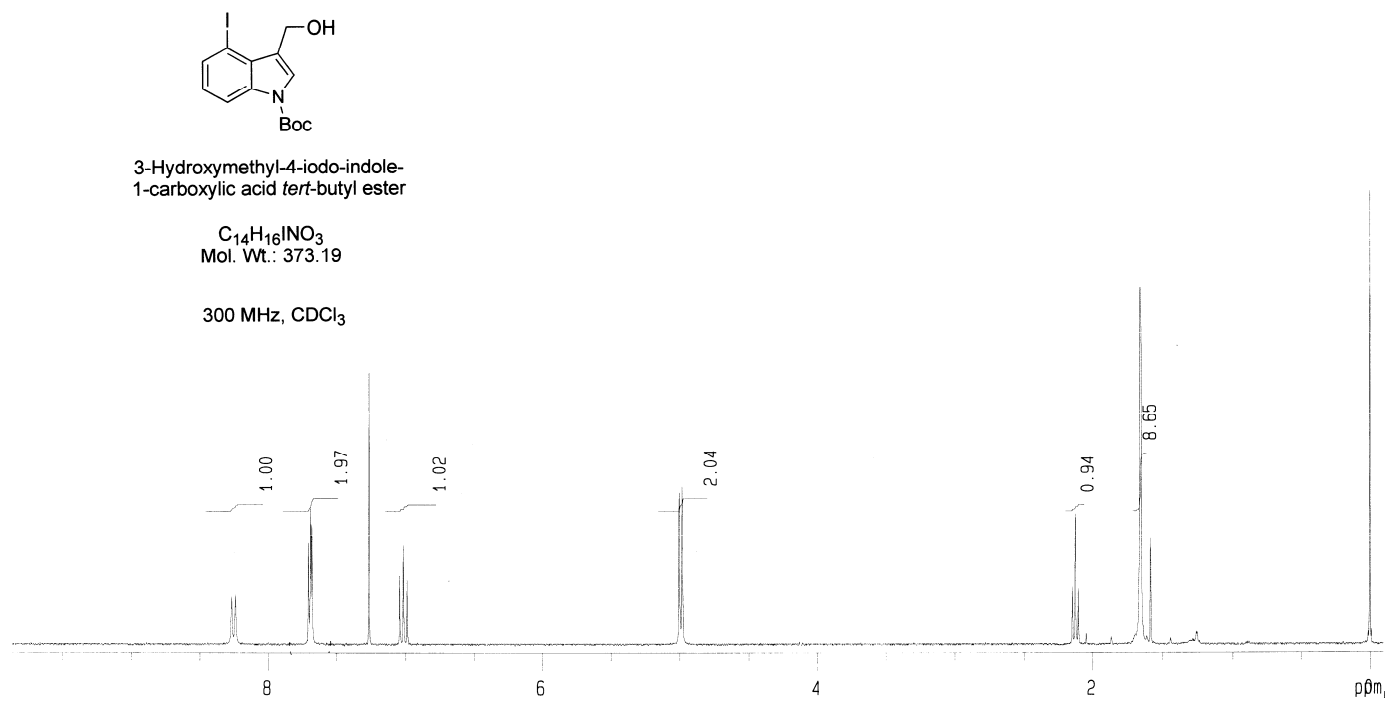

${ }^{13} \mathrm{C}$ NMR (100 MHz, $\mathrm{CDCl}_{3}$ )

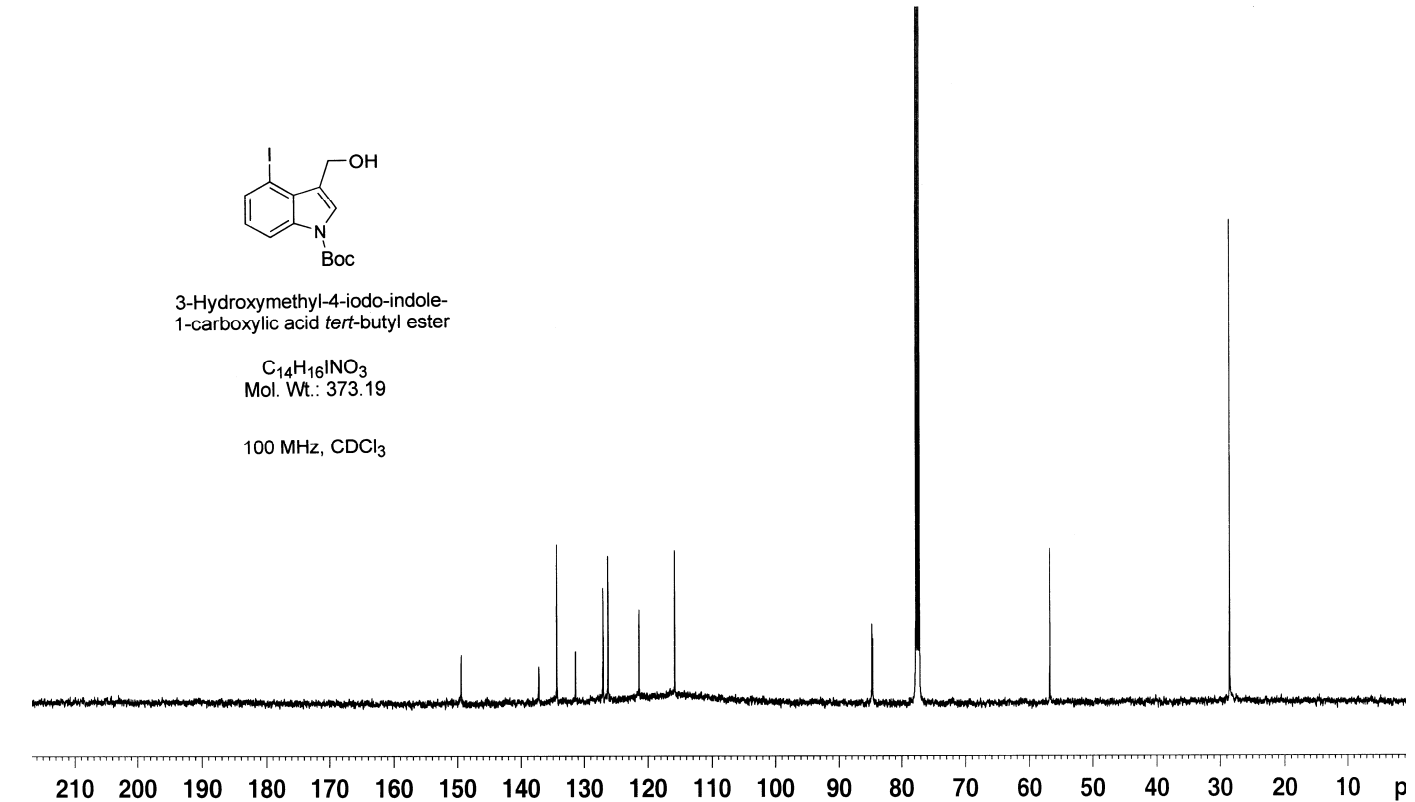




\section{Compound 13}

${ }^{1} \mathrm{H}$ NMR $\left(300 \mathrm{MHz}, \mathrm{C}_{6} \mathrm{D}_{6}\right)$

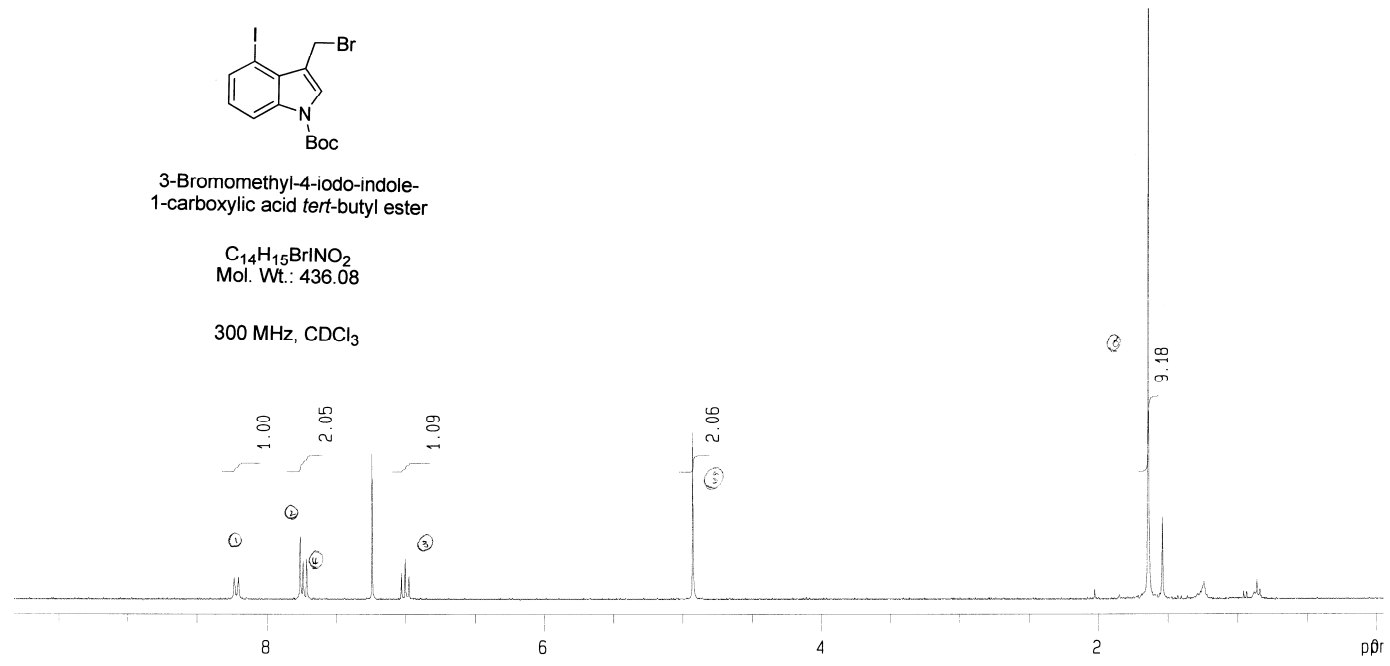

${ }^{13} \mathrm{C} \mathrm{NM}\left(75 \mathrm{MHz}, \mathrm{C}_{6} \mathrm{D}_{6}\right)$

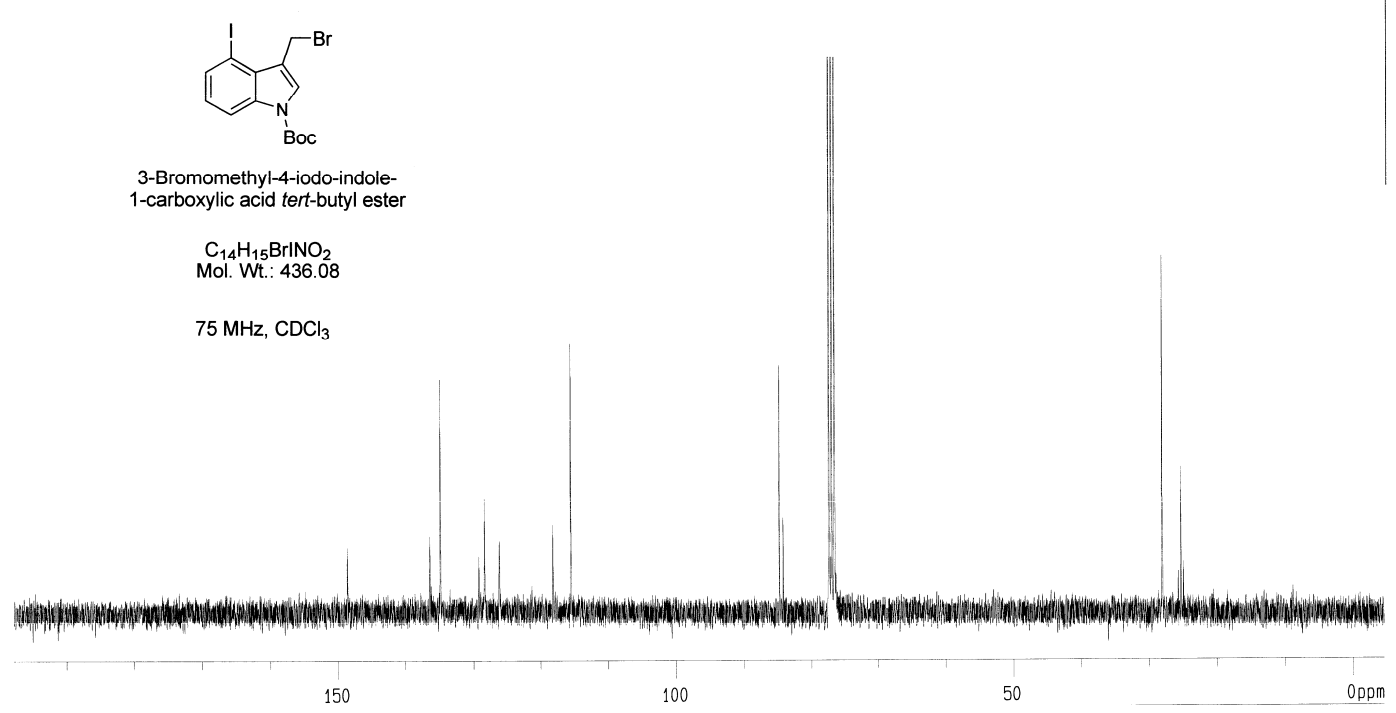




\section{Compound 14}

${ }^{1} \mathrm{H}$ NMR (300 MHz, $\mathrm{C}_{6} \mathrm{D}_{6}$ )
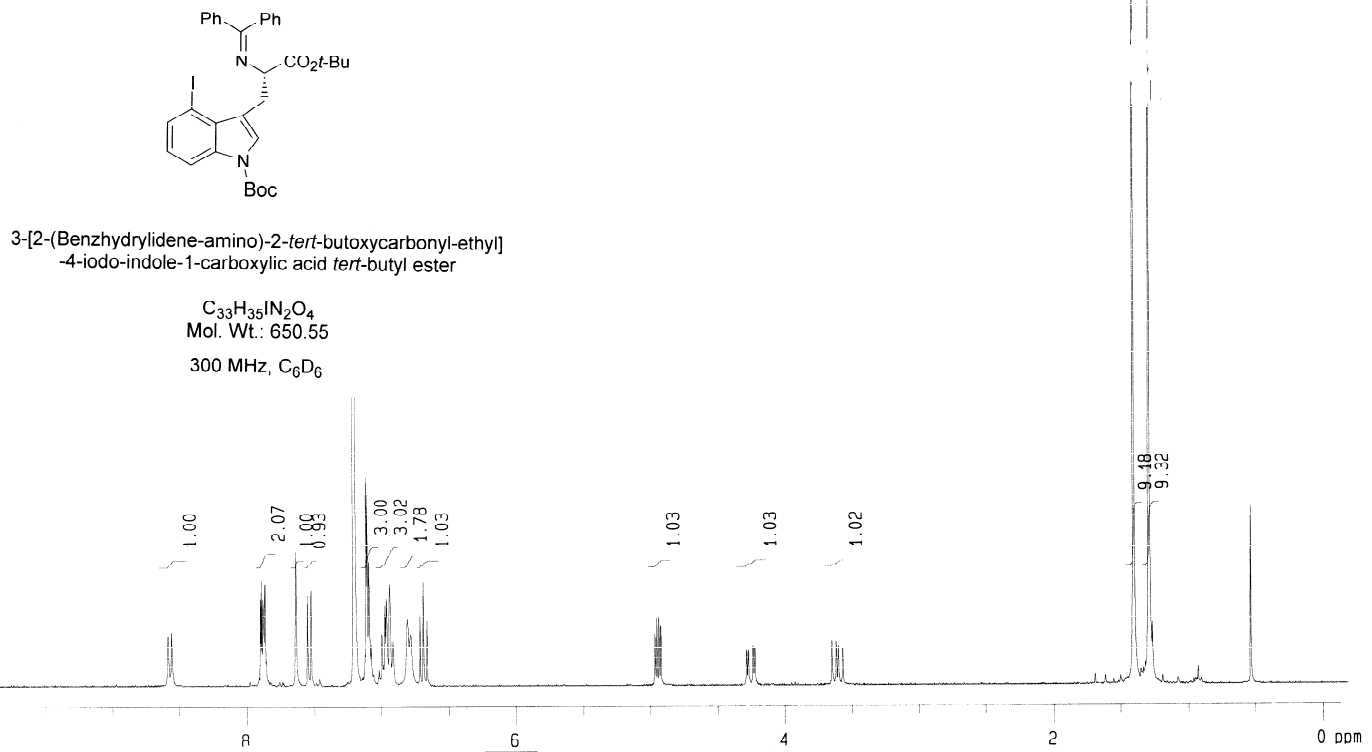

${ }^{13} \mathrm{C}$ NMR (100 MHz, $\left.\mathrm{C}_{6} \mathrm{D}_{6}\right)$

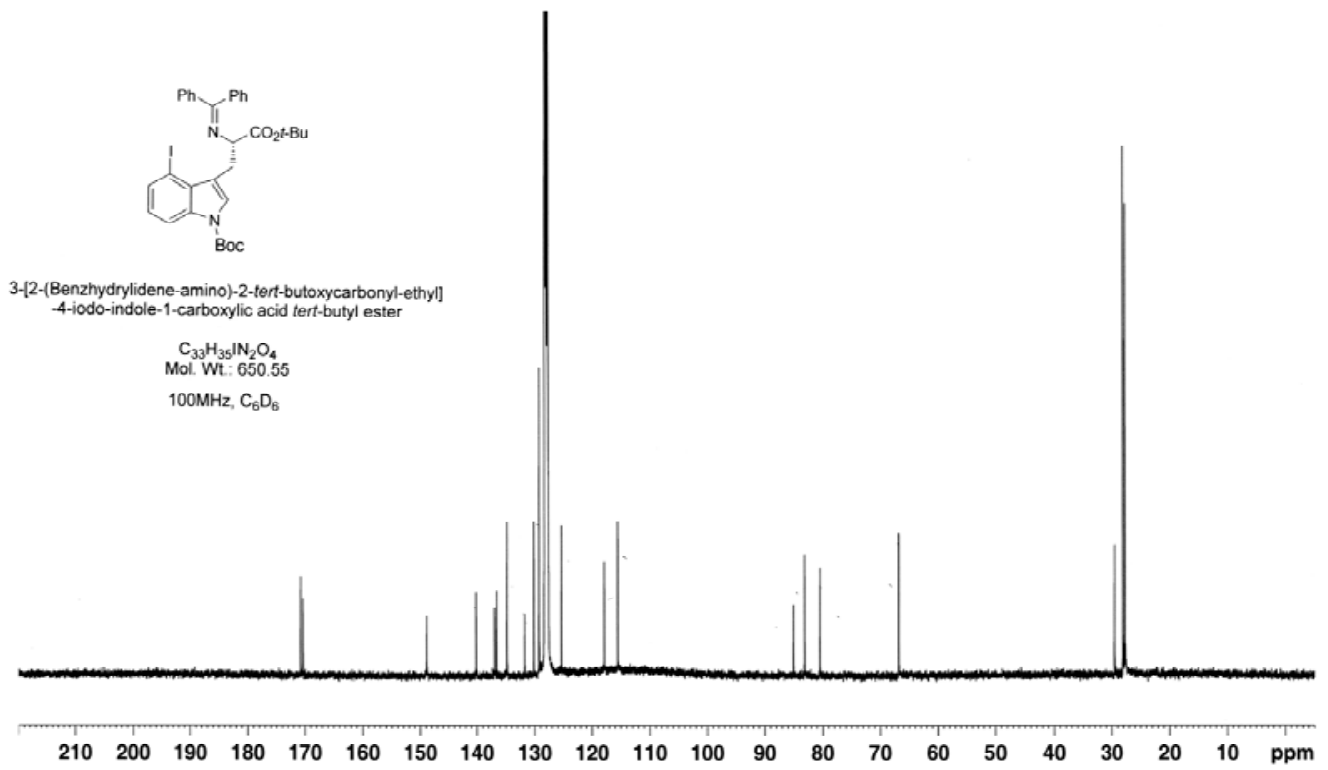




\section{Evaluation of enantiomeric excess of compound 14 by HPLC}
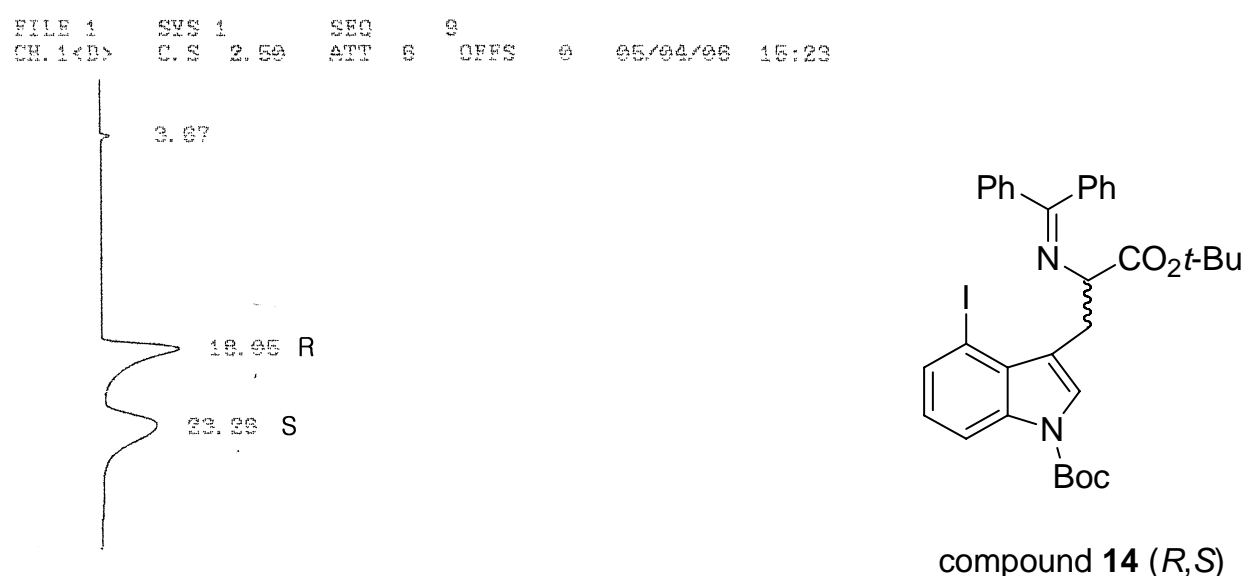

compound $14(R, S)$

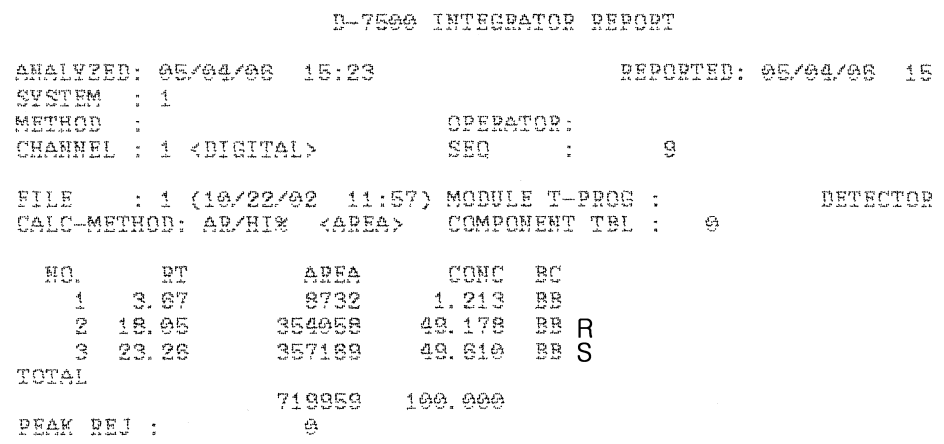

Chiralcel OD, hexanes:2-propanol $=500: 1$, flow rate $=1.0 \mathrm{~mL} / \mathrm{min}, 23{ }^{\circ} \mathrm{C}, \lambda=254 \mathrm{~nm}$, retention times; $R 18.5 \mathrm{~min}, S 23.2 \mathrm{~min}$. 


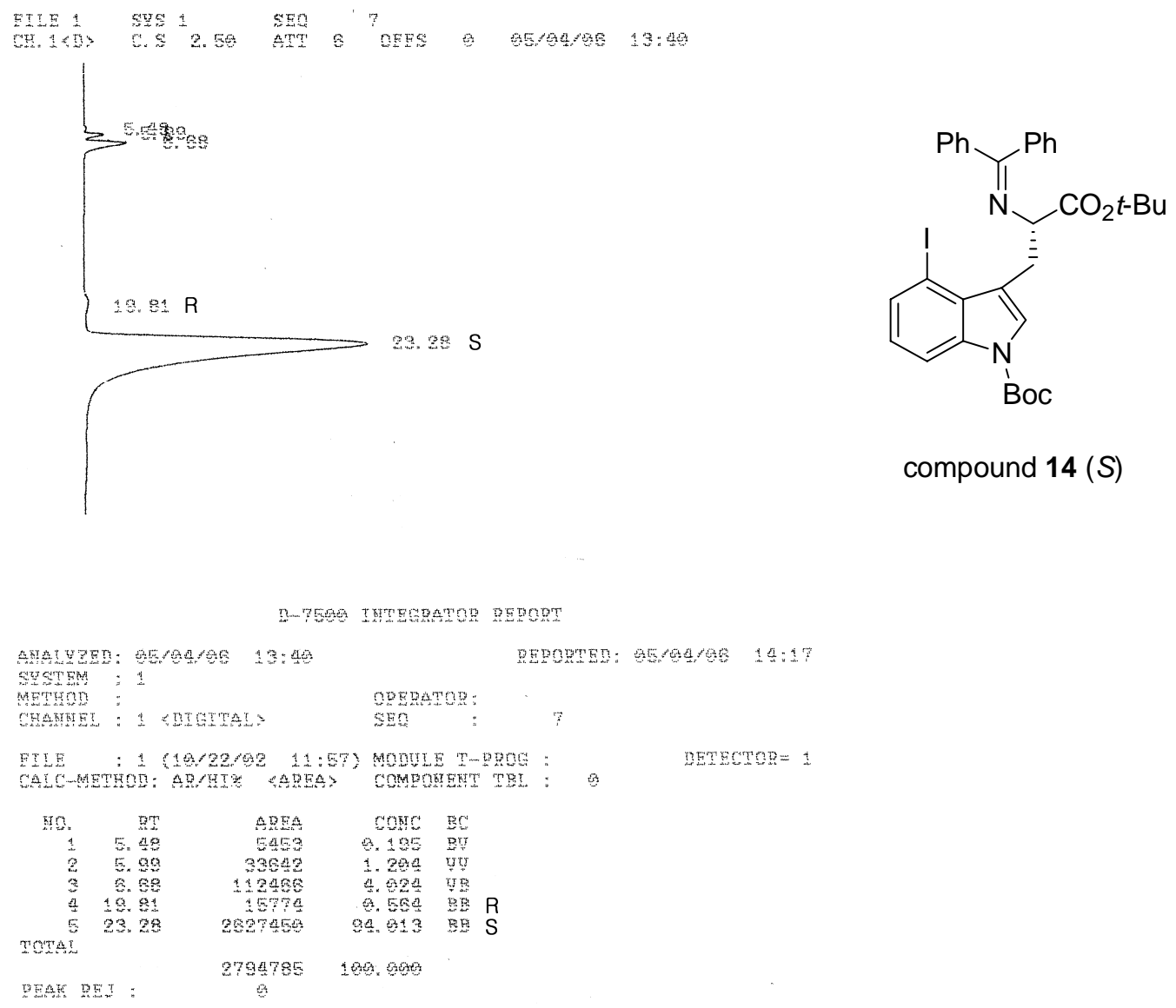

Chiralcel OD, hexanes:2-propanol $=500: 1$, flow rate $=1.0 \mathrm{~mL} / \mathrm{min}, 23{ }^{\circ} \mathrm{C}, \lambda=254 \mathrm{~nm}$, retention times; $R$ (minor) 19.0 min, $\mathrm{S}$ (major) $23.3 \mathrm{~min}, 99 \%$ ee. 


\section{Compound 15}

${ }^{1} \mathrm{H}$ NMR (300 MHz, $\mathrm{C}_{6} \mathrm{D}_{6}$ )

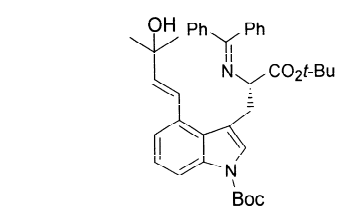

3-[2-(Benzhydrylidene-amino)-2-tert-butoxycarbony ethy!l]-4-(3-hydroxy-3-methy!-but-1-eny!)-indole 1 -carboxylic acid tert-butyl ester

$\mathrm{C}_{38} \mathrm{H}_{44} \mathrm{~N}_{2} \mathrm{O}_{5}$
$\mathrm{Mnl} \mathrm{Wt}_{4} \cdot 608.77$ $\mathrm{Mol} \mathrm{Wt}^{\circ} \cdot 608.77$

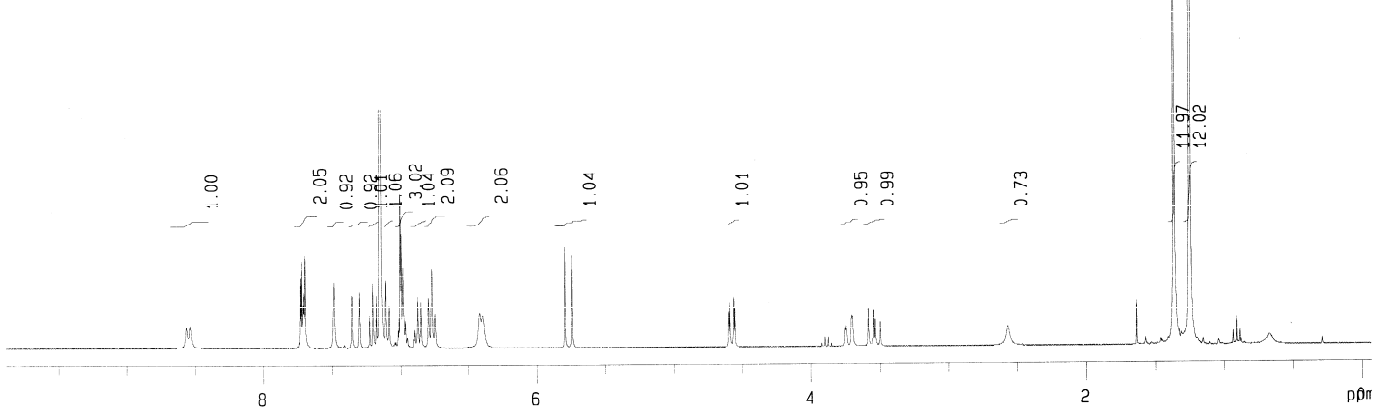

${ }^{13} \mathrm{C}$ NMR $\left(75 \mathrm{MHz}, \mathrm{C}_{6} \mathrm{D}_{6}\right)$

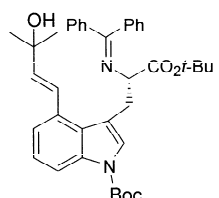

3-[2-(Benzhydrylidene-amino)-2-tert-butoxycarbony -ethyl!-4-(3-hydroxy-3-methyl-but-1-enyl)-indole 1 -carboxylic acid tert-butyl ester $\mathrm{C}_{38} \mathrm{H}_{44} \mathrm{~N}_{2} \mathrm{O}_{5}$
Mol. Wt.: 608.77 $75 \mathrm{MHz}, \mathrm{C}_{6} \mathrm{D}_{6}$

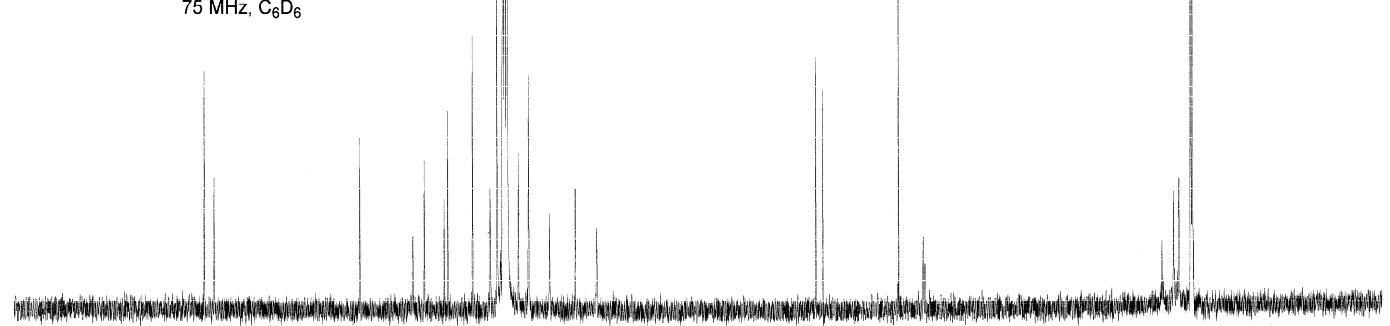
I H 
No racemization in Hack reaction was confirmed by chiral HPLC analysis.

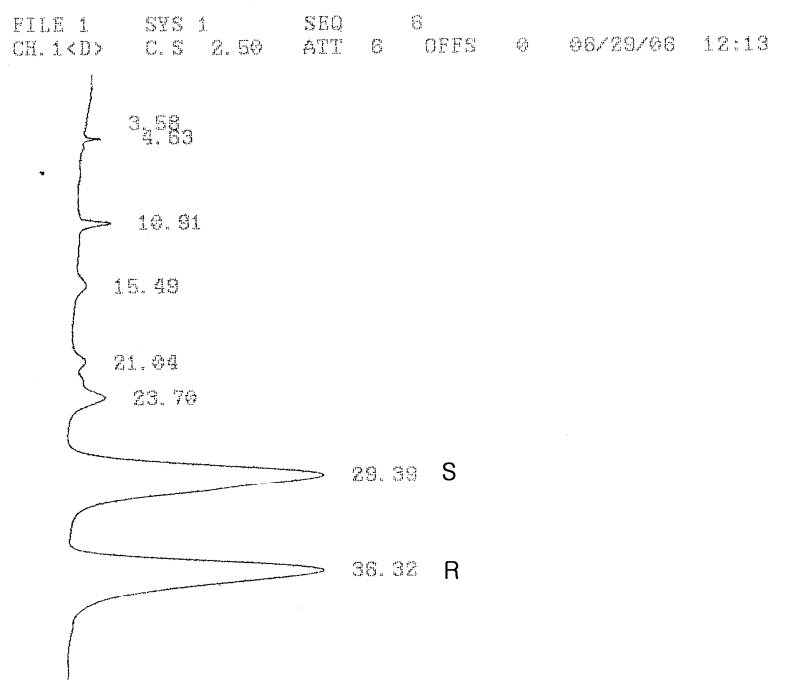

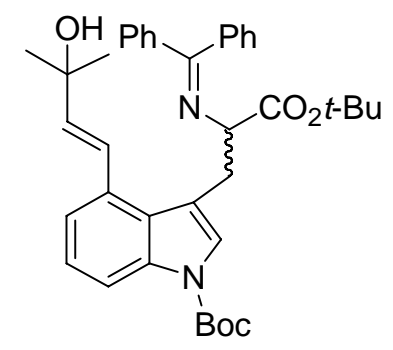

compound $15(R, S)$

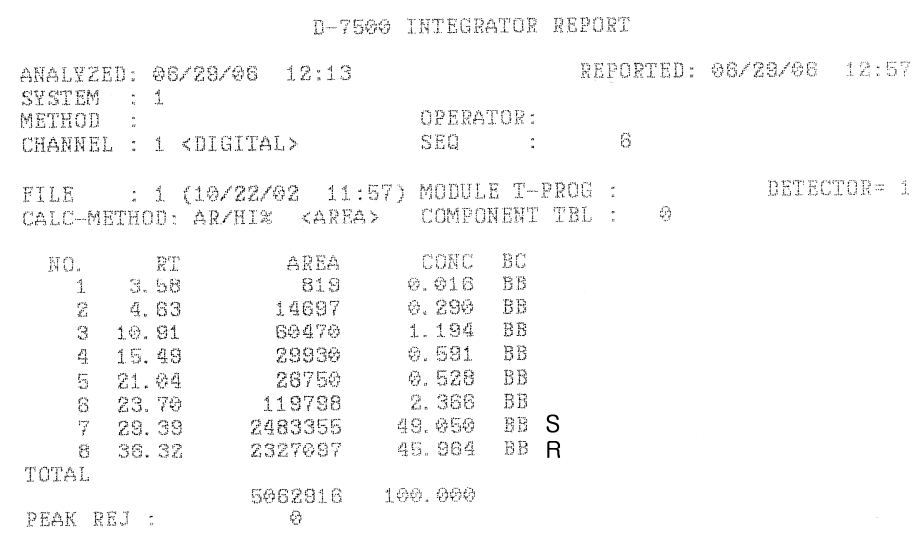

Chiralcel OD, hexanes:2-propanol $=99: 1$, flow rate $=1.0 \mathrm{~mL} / \mathrm{min}, 23{ }^{\circ} \mathrm{C}, \lambda=254 \mathrm{~nm}$, retention times; $S 29.4 \mathrm{~min}, R 36.3 \mathrm{~min}$. 


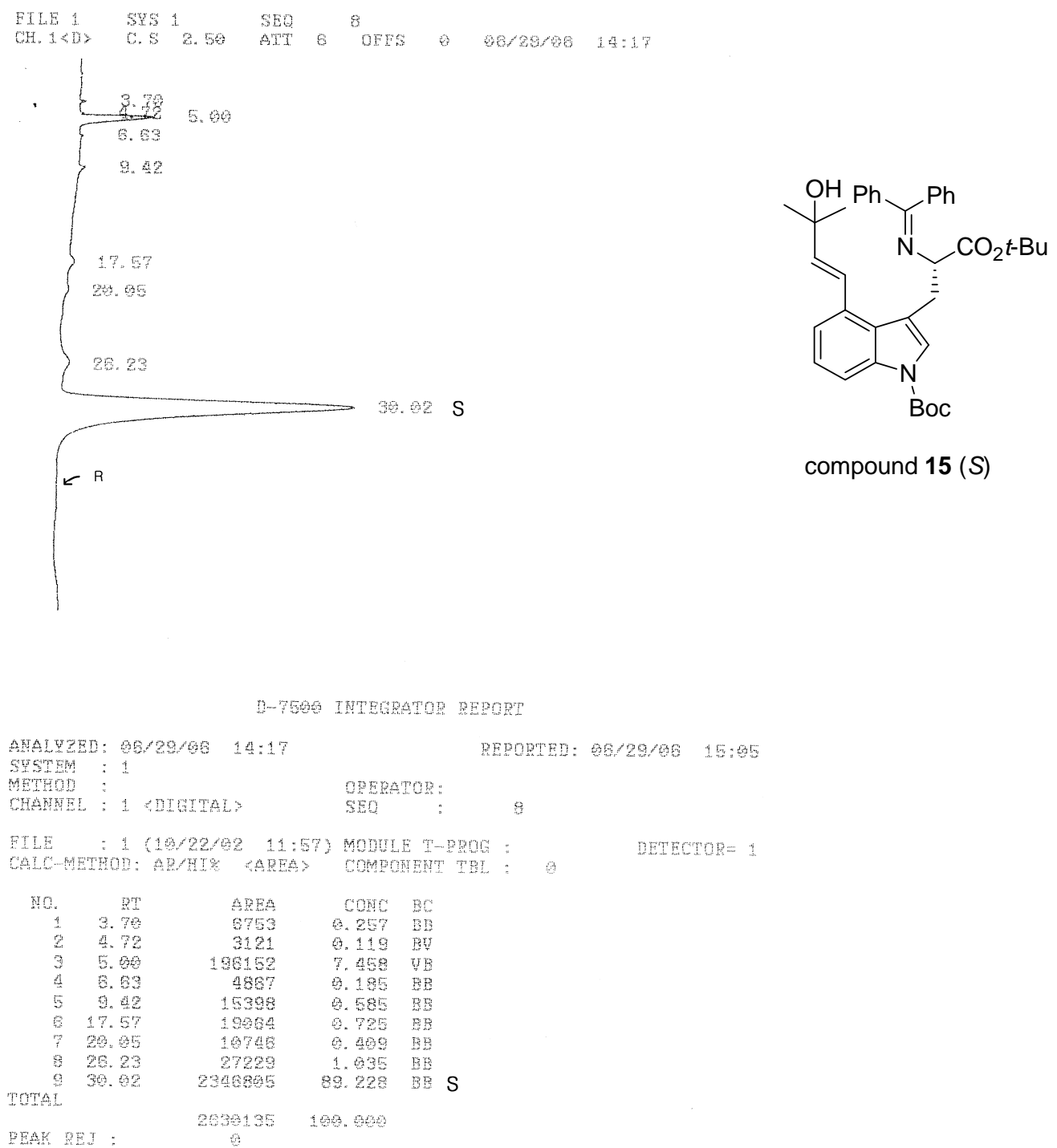

Chiralcel OD, hexanes:2-propanol $=99: 1$, flow rate $=1.0 \mathrm{~mL} / \mathrm{min}, 23{ }^{\circ} \mathrm{C}, \lambda=254 \mathrm{~nm}$, retention times; $\mathrm{S}$ (major) $30.0 \mathrm{~min}, R$ (not identify), $99 \%$ ee. 


\section{Compound 16}

${ }^{1} \mathrm{H}$ NMR $\left(300 \mathrm{MHz}, \mathrm{CD}_{3} \mathrm{OD}\right)$
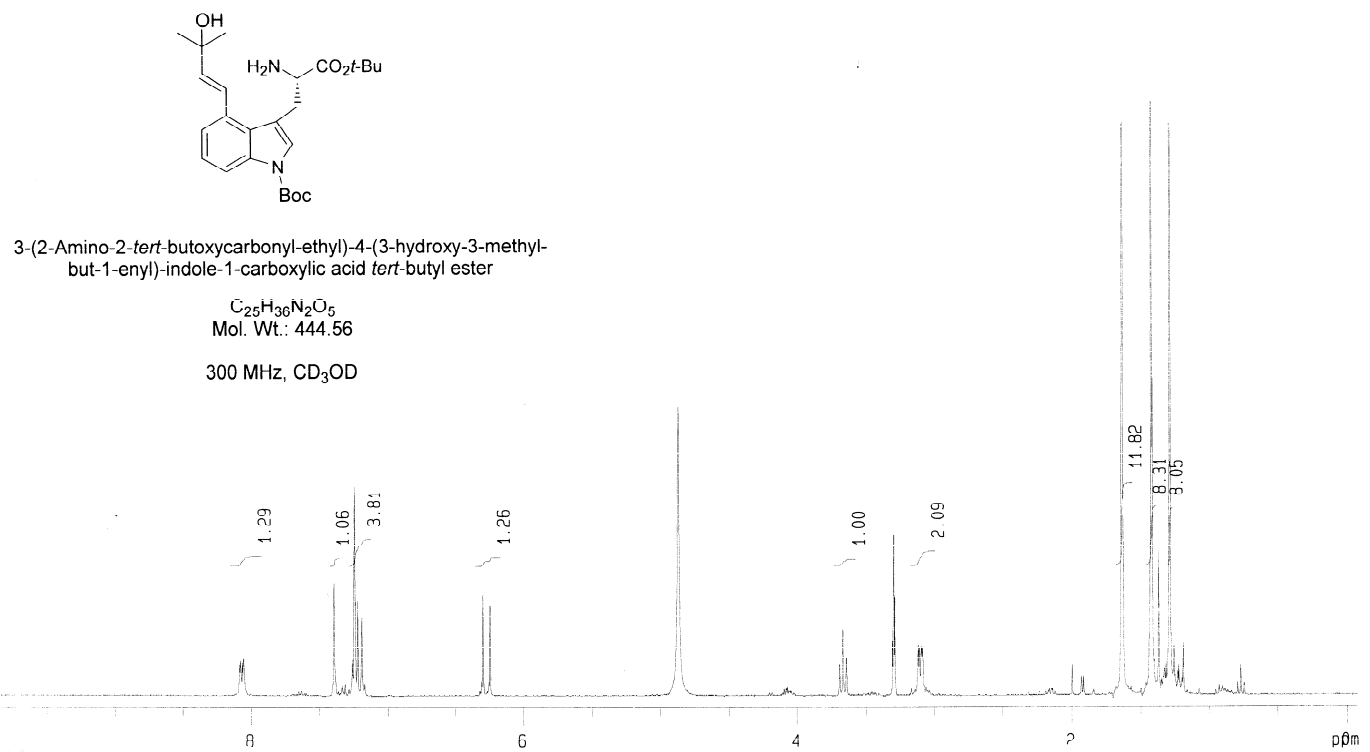

\section{${ }^{13} \mathrm{C}$ NMR $\left(100 \mathrm{MHz}, \mathrm{CD}_{3} \mathrm{OD}\right)$}
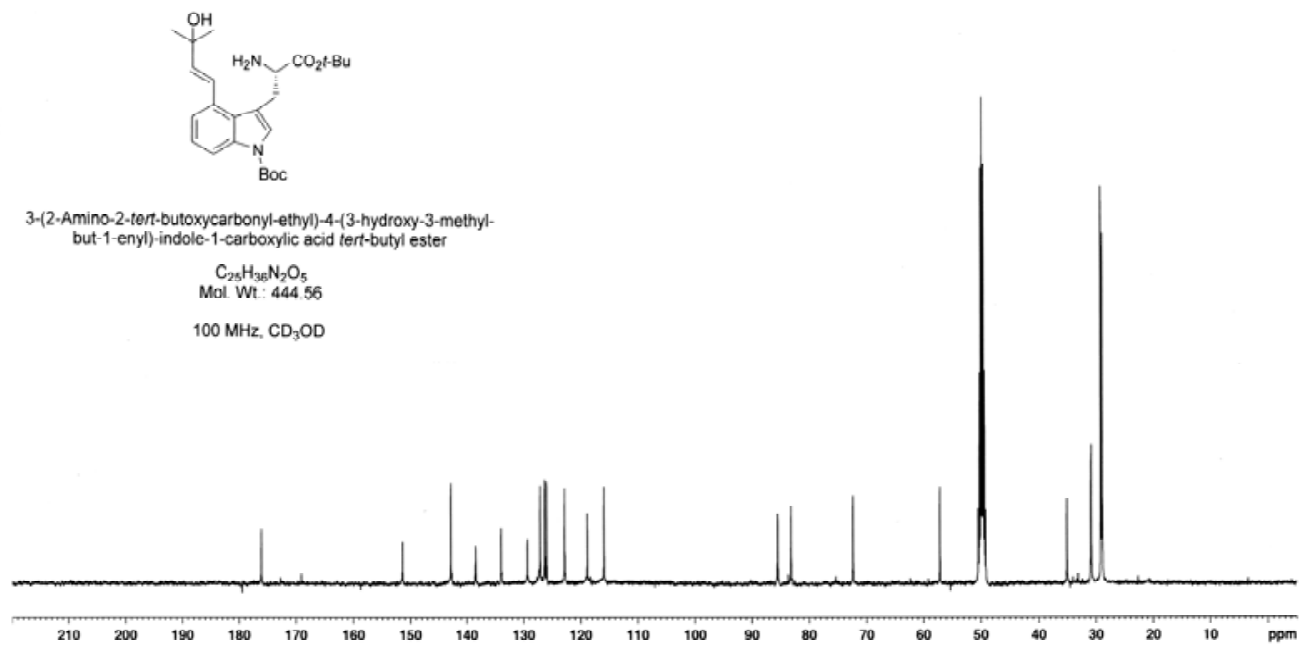


\section{Compound 17}

${ }^{1} \mathrm{H}$ NMR (300 MHz, $\left.\mathrm{CD}_{3} \mathrm{OD}\right)$

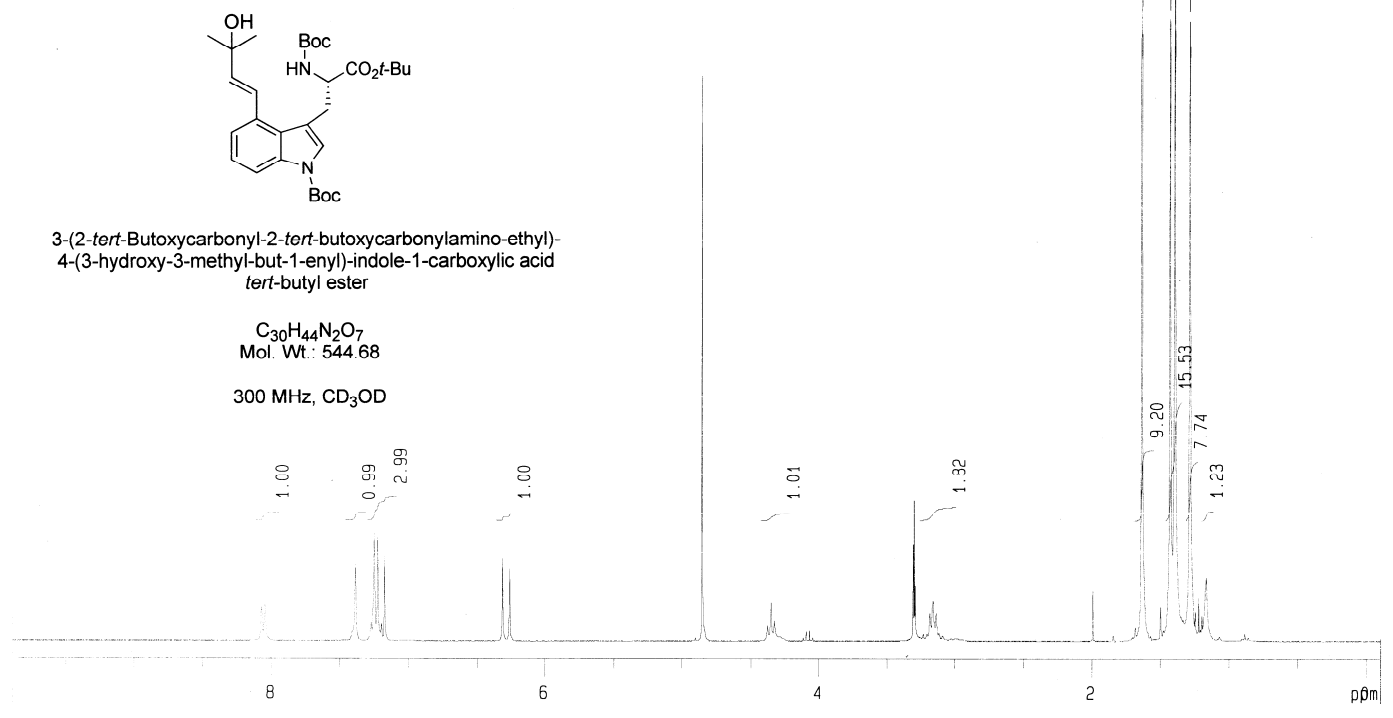

${ }^{13} \mathrm{C}$ NMR (100 MHz, $\left.\mathrm{CD}_{3} \mathrm{OD}\right)$

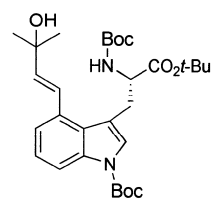

3-(2-tert-Butoxycarbonyl-2-tert-butoxycarbonylamino-ethyl)4-(3-hydroxy-3-methyl-but-1-enyl)-indole-1-carboxylic acid
tert-butyl ester

$\mathrm{C}_{30} \mathrm{H}_{44} \mathrm{~N}_{2} \mathrm{O}_{7}$
Mol. Wt.: 544.68

$100 \mathrm{MHz}, \mathrm{CD}_{3} \mathrm{OD}$
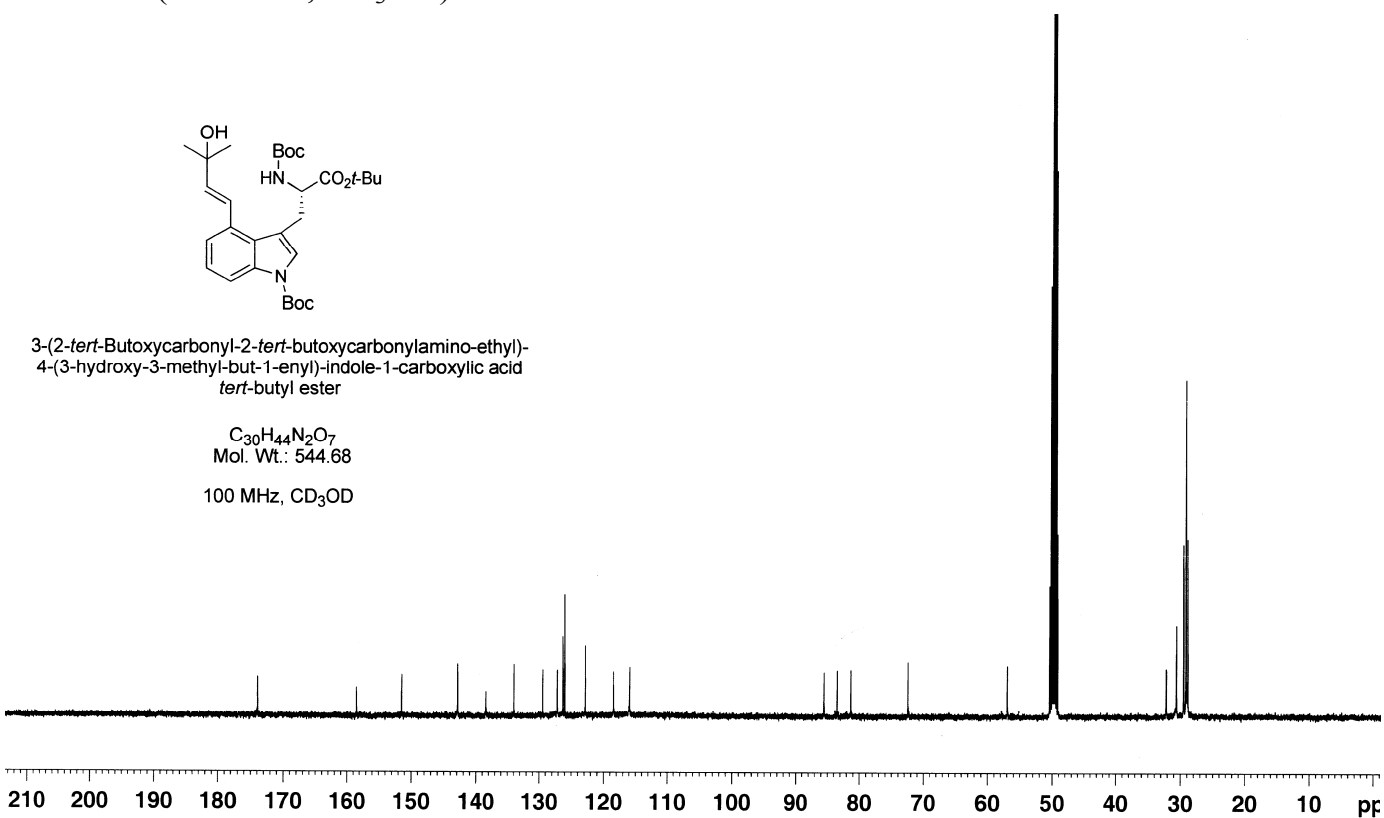


\section{Compound cis-1}

${ }^{1} \mathrm{H}$ NMR $\left(300 \mathrm{MHz}, \mathrm{CD}_{3} \mathrm{OD}\right)$

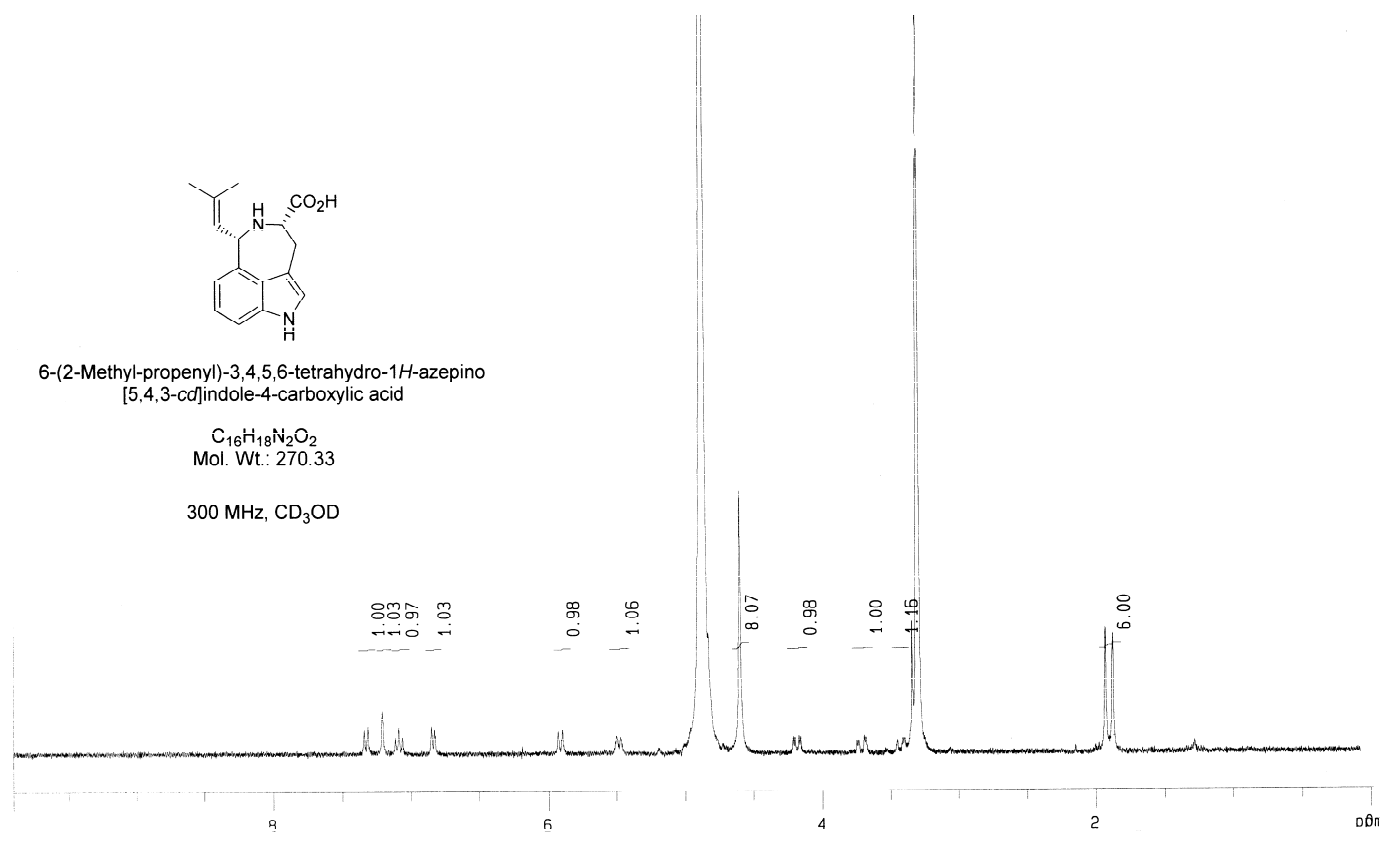


Compound trans-1

${ }^{1} \mathrm{H}$ NMR (300 MHz, $\left.\mathrm{CD}_{3} \mathrm{OD}\right)$

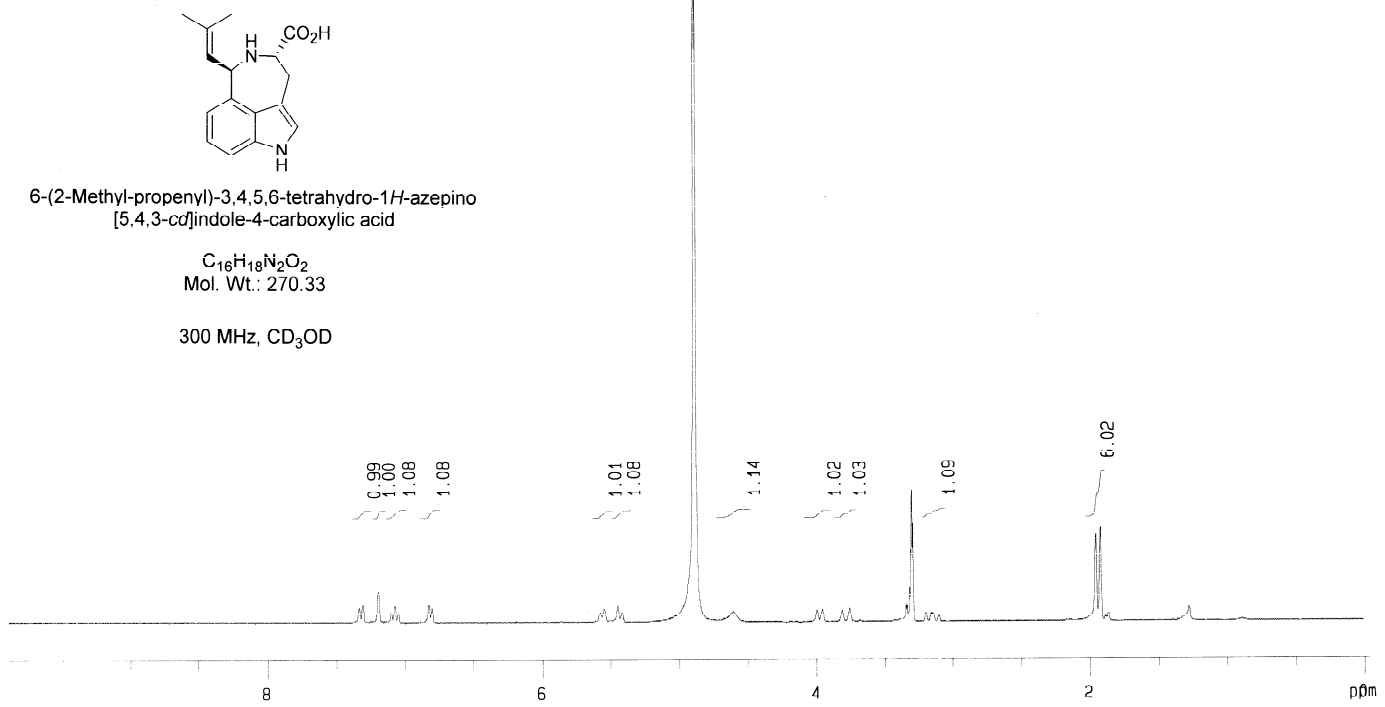

${ }^{13} \mathrm{C}$ NMR (75 MHz, $\left.\mathrm{CD}_{3} \mathrm{OD}\right)$

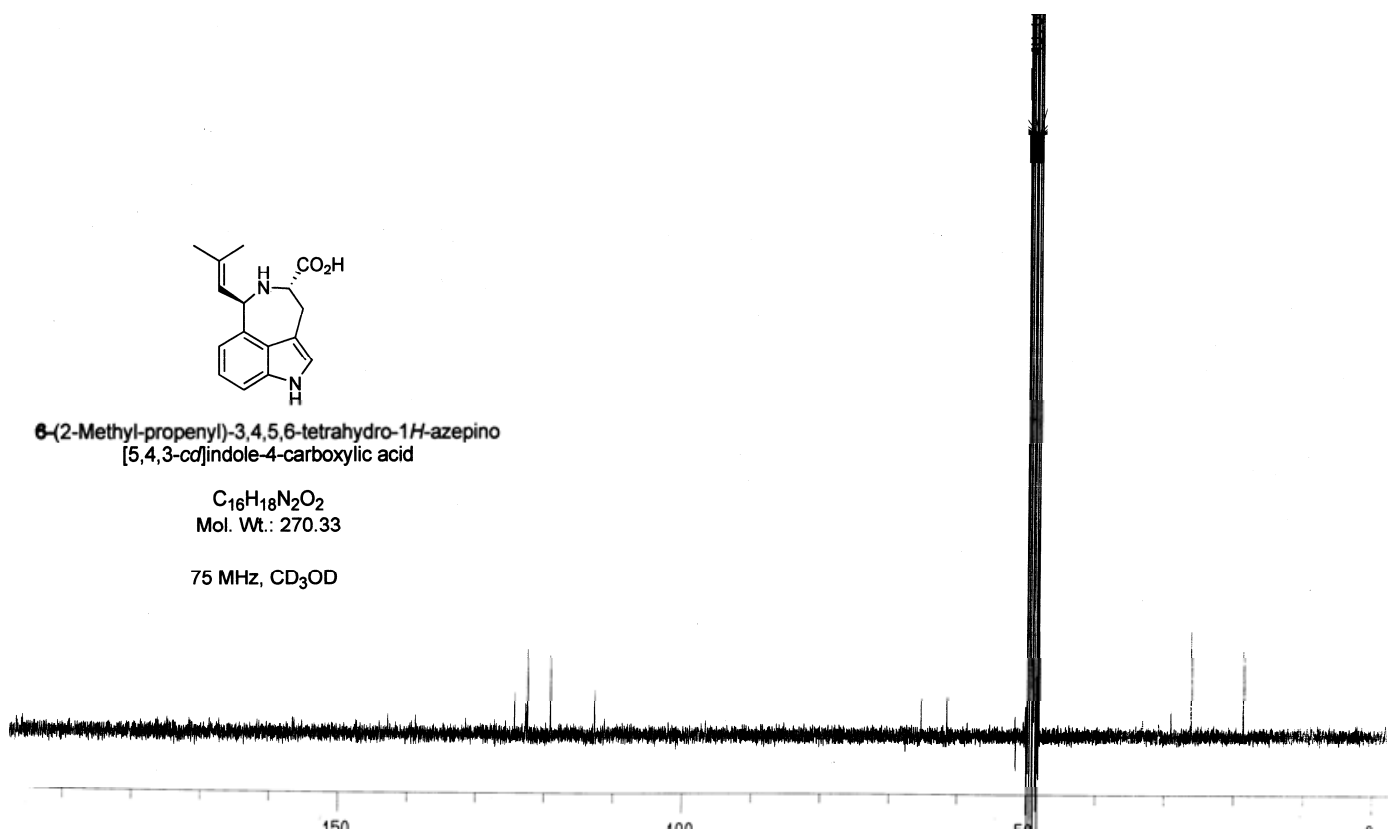


Compound 19a

${ }^{1} \mathrm{H}$ NMR $\left(300 \mathrm{MHz}, \mathrm{CD}_{3} \mathrm{OD}\right)$

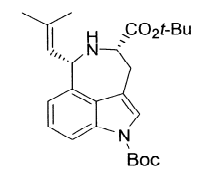

6-(2-Methyl-propenyl)-3,4,5,6-tetrahydro-azepino[5,4,3-cd] indole-1,4-dicarboxylic acid di-tert-butyl ester

$$
\begin{gathered}
\mathrm{C}_{25} \mathrm{H}_{34} \mathrm{~N}_{2} \mathrm{O}_{4} \\
\text { Mol. Wt.: } 426.55
\end{gathered}
$$

$300 \mathrm{MHz}, \mathrm{CD}_{3} \mathrm{OD}$

$$
\text { 음 }=
$$

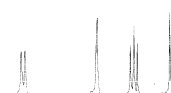

8

${ }^{13} \mathrm{C}$ NMR (100 MHz, $\left.\mathrm{CD}_{3} \mathrm{OD}\right)$

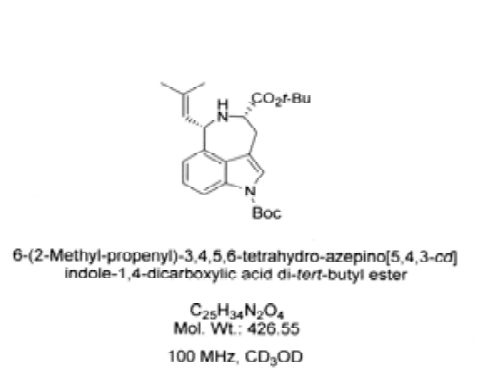

$100 \mathrm{MHz}, \mathrm{CD}_{3} \mathrm{OD}$ 


\section{Compound 19b}

${ }^{1} \mathrm{H}$ NMR $\left(300 \mathrm{MHz}, \mathrm{CD}_{3} \mathrm{OD}\right)$

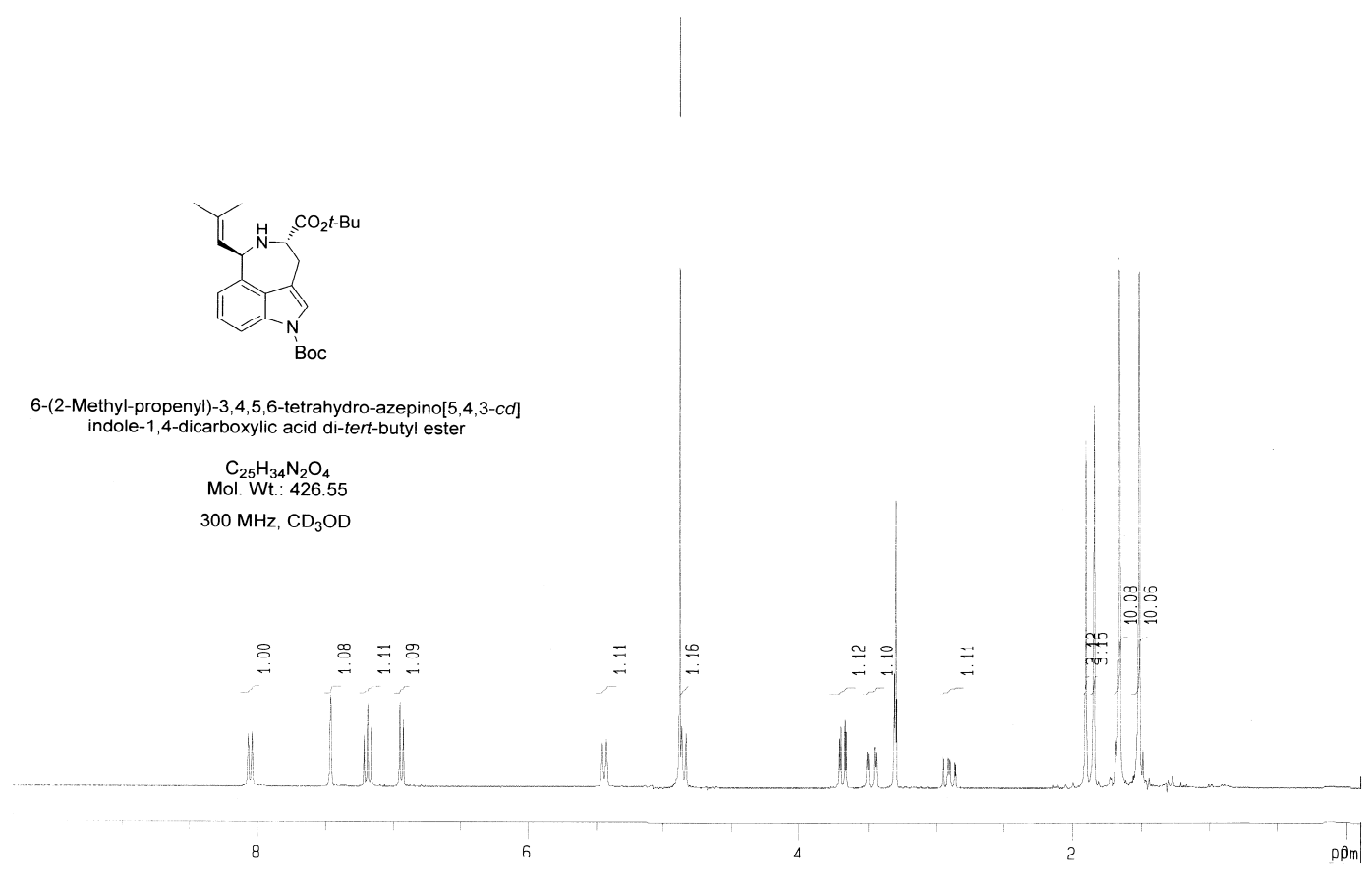

\section{${ }^{13} \mathrm{C}$ NMR (75 MHz, $\left.\mathrm{CD}_{3} \mathrm{OD}\right)$}
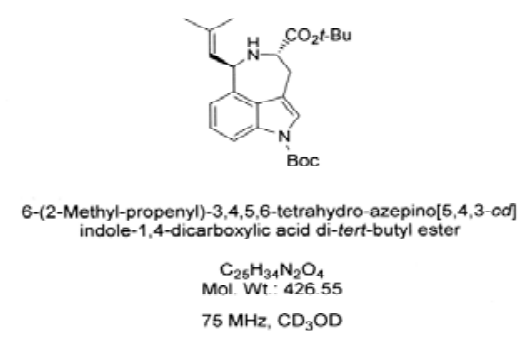

S28 